Document downloaded from:

http://hdl.handle.net/10251/150301

This paper must be cited as:

Albanese, AA.; Jornet Casanova, D. (2016). Dissipative operators and additive perturbations in locally convex spaces. Mathematische Nachrichten. 289(8-9):920-949.

https://doi.org/10.1002/mana.201500150

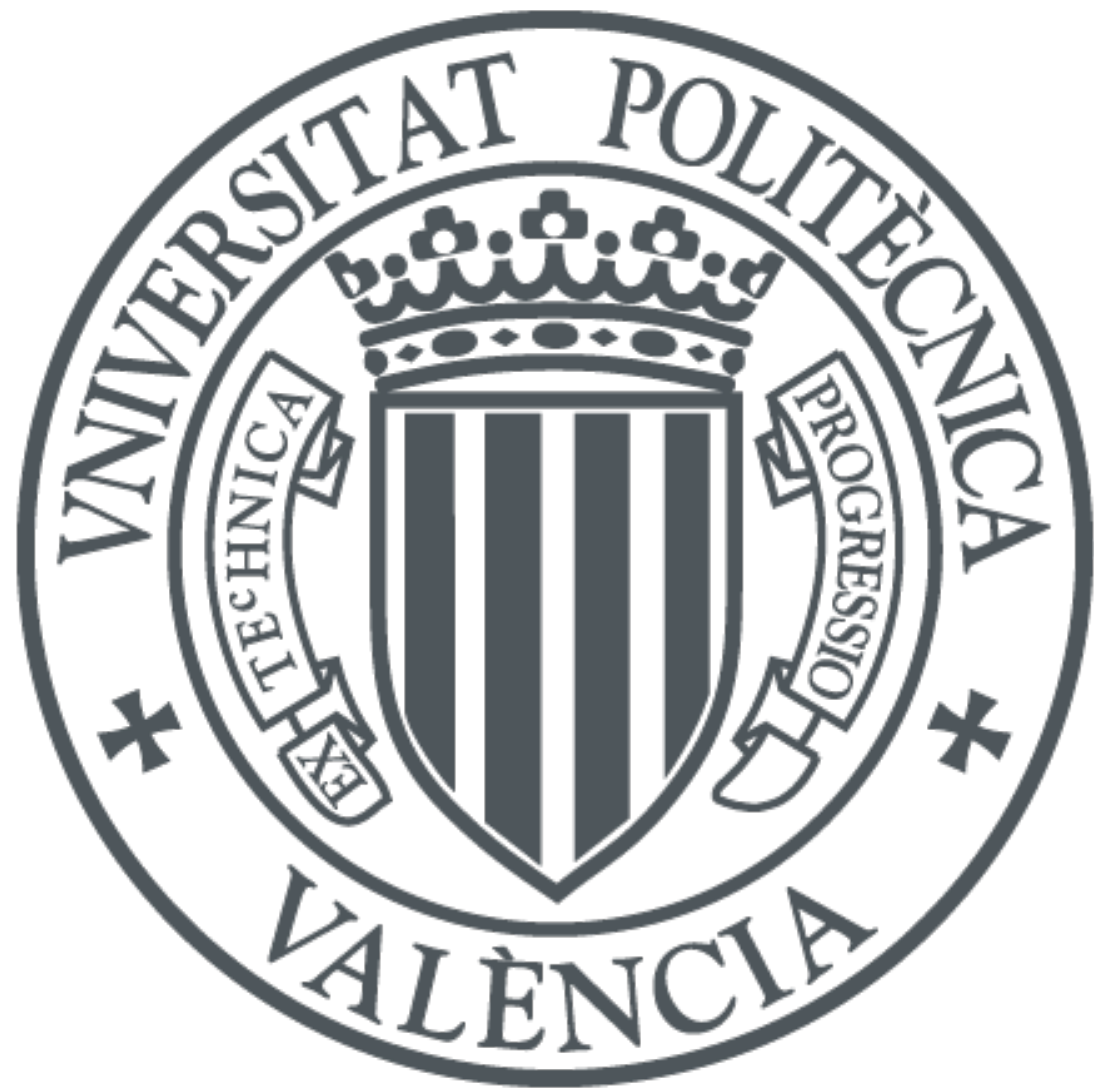

The final publication is available at

https://doi.org/10.1002/mana.201500150

Copyright John Wiley \& Sons

Additional Information

"This is the peer reviewed version of the following article: Albanese, Angela A., and David Jornet. 2015. Dissipative Operators and Additive Perturbations in Locally Convex Spaces. Mathematische Nachrichten 289 (8 9). Wiley: 920 49. doi:10.1002/mana.201500150, which has been published in final form at https://doi.org/10.1002/mana.201500150. This article may be used for non-commercial purposes in accordance with Wiley Terms and Conditions for Self-Archiving." 


\title{
DISSIPATIVE OPERATORS AND ADDITIVE PERTURBATIONS IN LOCALLY CONVEX SPACES
}

\author{
ANGELA A. ALBANESE AND DAVID JORNET
}

\author{
Dedicated to our dear friend José Bonet on the occasion of his 60th birthday
}

\begin{abstract}
Let $(A, D(A))$ be a densely defined operator on a Banach space $X$. Characterizations of when $(A, D(A))$ generates a (an equicontinuous) $C_{0}$-semigroup on $X$ are known. For instance, a famous result of Lumer and Phillips states that it is so if and only if $(A, D(A))$ is dissipative and $\overline{\operatorname{rg}(\lambda I-A)}=X$ for some $\lambda>0$. There exists also a rich amount of Banach space results concerning perturbations of dissipative operators. In a recent paper Tyran-Kamińska provides perturbation criteria of dissipative operators in terms of ergodic properties.

These results, and others, are shown to remain valid in the setting of general non-normable lcHs'. Applications of the results to concrete examples of operators on function spaces are also presented.
\end{abstract}

\section{Introduction}

The theory of unbounded operators is an important and intensively studied topic in the context of $C_{0}$-semigroups of continuous linear operators acting on Banach spaces. In the literature there exists a vast amount of results covering various types of generation, perturbation, approximation, asymptotics on different classes of Banach and Hilbert spaces. We refer to [13, 25] and the references therein. Various notions and results from the theory of unbounded operators on Banach spaces had been successfully generalized to the setting of locally convex spaces; see [19], [23] and [28] for a sample. Hovewer, there is an essential difference between the Banach space case and the locally convex space case. For instance, in the Banach space setting the resolvent set $\rho(A)$ of the infinitesimal generator $(A, D(A))$ of a $C_{0}$-semigroup is always not empty and open; in the locally convex space setting it can happen that $\rho(A)=\emptyset$; see [20]. Actually, leaving the Banach space setting, many results of this theory do not carry over to general locally convex spaces; the results do not even extend to general Fréchet spaces. On the other hand, modern analysis occurs in non-normable locally convex spaces. So, there is an interest to understand what is possible to extend from Banach space setting to this more general setting. This analysis has been carried out in recent years; see, f.i., $[3,4,5,7,12,14,15,16,27]$ and the references therein. For instance, Domański and Langenbruch [12] introduce a general notion of resolvent for operators on locally convex spaces to provide a complete solution of the abstract Cauchy problem for operator valued Laplace distributions or hyperfunctions on complete ultrabornological locally convex spaces. In a recent paper Jacob, Wegner and Wintermayr [16] prove a Desch-Schappacher perturbation theorem for locally equicontinuous $C_{0}$-semigroups of continuous linear operators acting on sequentially complete locally convex spaces. On the other hand, Albanese, Bonet and Ricker [7] show that the uniform mean ergodicity of a $C_{0}$-semigroup $(T(t))_{t \geq 0}$ on a locally convex Hausdorff space $X$ is equivalent to the closedness of the range of its infinitesimal generator whenever $X$ belongs to a class of Fréchet spaces, the so-called quojection Fréchet spaces, as it happens in Banach space case. They show also that this characterization fails in the setting of general Fréchet spaces; see also [5] for more information.

The purpose of the present paper is to study when some known Banach space results concerning dissipative operators and their perturbation carry over to general locally convex Hausdorff spaces.

Key words and phrases. Equicontinuous semigroup, dissipative operator, additive perturbation, (uniformly) mean ergodic operator, quasi-Montel operator, locally convex space.

Mathematics Subject Classification 2010: Primary 47B44, 47A55, 46A99; Secondary 47D03, 47A35. 
To this aim, in Section 3 we develop an introductory theory of closable operators and dissipative operators on locally convex spaces. In particular, we show that the classical result of Lumer and Phillips remains valid in the setting of non-normable complete locally convex spaces; see Theorem 3.13. In the end of Section 3 we also collect some simple conditions for an operator to generate an equicontinuous $C_{0}$-semigroup. In Section 4 , we establish additive perturbation results of dissipative operators on some classes of locally convex spaces; see Theorems 4.10 and 4.12 . To this end, we first adapt the notion of subdifferential in this setting and we then collect some relative properties. In Section 4 applications of the perturbation results to concrete examples of operators on function spaces are also presented. The results from Sections 3 and 4 together with recent developments in ergodic theory in locally convex Hausdorff spaces investigated in $[1,2,6,8]$ allow us to extend from the Banach space case to locally convex space case the results on additive perturbations, due to Tyran-Kamińska [26], in terms of ergodic properties; see Theorems 5.1, 5.2, 5.4 and 5.5 of Section 5. For instance, in Section 5 we show that if $(A, D(A))$ and $(B, D(B))$ are two operators acting on a quojection Fréchet space $X$ such that $(A+B, D(A))$ is dissipative and $B R(\lambda, A)$ is uniformly mean ergodic for some $\lambda>0$, then $(A+B, D(A))$ generates an equicontinuous $C_{0}$-semigroup on $X$; see Theorem 5.4. But, we also show that this result fails in the setting of general Fréchet spaces; see Example 5.7.

\section{Preliminaries and main notation}

Let $X$ be a locally convex Hausdorff space (briefly, lcHs) and $\Gamma_{X}$ a system of continuous seminorms determining the locally convex topology (briefly, lc-topology) of $X$. The strong operator topology $\tau_{s}$ in the space $\mathcal{L}(X)$ of all continuous linear operators from $X$ into itself is determined by the family of seminorms $q_{x}(S):=q(S x)$, for $S \in \mathcal{L}(X)$, for each $x \in X$ and $q \in \Gamma_{X}$; in such a case we write $\mathcal{L}_{s}(X)$. Denote by $\mathcal{B}(X)$ the collection of all bounded subsets of $X$. The topology $\tau_{b}$ of uniform convergence on bounded sets is defined in $\mathcal{L}(X)$ via the seminorms $q_{B}(S):=\sup _{x \in B} q(S x)$, for $S \in \mathcal{L}(X)$, for each $B \in \mathcal{B}(X)$ and $q \in \Gamma_{X}$; in this case we write $\mathcal{L}_{b}(X)$. In case $X$ is a Banach space, $\tau_{b}$ is the operator norm topology in $\mathcal{L}(X)$. If $\Gamma_{X}$ is countable and $X$ is complete, then $X$ is called a Fréchet space. The identity operator on a lcHs $X$ is denoted by $I$.

By $X_{\sigma}$ we denote $X$ equipped with its weak topology $\sigma\left(X, X^{\prime}\right)$, where $X^{\prime}$ is the topological dual space of $X$. The strong topology in $X$ (resp. $X^{\prime}$ ) is denoted by $\beta\left(X, X^{\prime}\right)$ (resp. $\beta\left(X^{\prime}, X\right)$ ) and we write $X_{\beta}$ (resp. $\left.X_{\beta}^{\prime}\right)$; see $[17, \S 21.2]$ for the definition. The strong dual space $\left(X_{\beta}^{\prime}\right)_{\beta}^{\prime}$ of $X_{\beta}^{\prime}$ is denoted simply by $X^{\prime \prime}$. By $X_{\sigma}^{\prime}$ we denote $X^{\prime}$ equipped with its weak-star topology $\sigma\left(X^{\prime}, X\right)$. Given $T \in \mathcal{L}(X)$, its dual operator $T^{\prime}: X^{\prime} \rightarrow X^{\prime}$ is defined by $\left\langle x, T^{\prime} x^{\prime}\right\rangle=\left\langle T x, x^{\prime}\right\rangle$ for all $x \in X, x^{\prime} \in X^{\prime}$. It is known that $T^{\prime} \in \mathcal{L}\left(X_{\sigma}^{\prime}\right)$ and $T^{\prime} \in \mathcal{L}\left(X_{\beta}^{\prime}\right),[18, \mathrm{p} .134]$.

Definition 2.1. Let $X$ be a lcHs and $(T(t))_{t \geq 0} \subseteq \mathcal{L}(X)$ be a 1-parameter family of operators.

We say that $(T(t))_{t \geq 0}$ is a semigroup if it satisfies

(i) $T(s) T(t)=T(s+t)$ for all $s, t \geq 0$, with $T(0)=I$.

A semigroup $(T(t))_{t \geq 0}$ is locally equicontinuous if, for fixed $K>0$, the set $\{T(t): 0 \leq t \leq K\}$ is equicontinuous, i.e., for every $p \in \Gamma_{X}$ there exist $q \in \Gamma_{X}$ and $M>0$ (depending on $p$ and $K$ ) such that

$$
p(T(t) x) \leq M q(x), \quad x \in X, t \in[0, K] .
$$

A semigroup $(T(t))_{t \geq 0}$ is said to be a $C_{0}$-semigroup if it satisfies

(ii) $\lim _{t \rightarrow 0^{+}} T(t)=I$ in $\mathcal{L}_{s}(X)$.

If the $C_{0}$-semigroup $(T(t))_{t \geq 0}$ satisfies the additional condition that

(iii) $\lim _{t \rightarrow t_{0}} T(t)=T\left(t_{0}\right)$ in $\mathcal{L}_{s}(X)$, for each $t_{0} \geq 0$,

then it is called a strongly continuous $C_{0}$-semigroup. 
A semigroup $(T(t))_{t \geq 0}$ is called exponentially equicontinuous if there exists $a \geq 0$ such that the semigroup $\left(e^{-a t} T(t)\right)_{t \geq 0}$ in $\mathcal{L}(X)$ is equicontinuous, i.e.,

$$
\forall p \in \Gamma_{X} \exists q \in \Gamma_{X}, M_{p}>0: p(T(t) x) \leq M_{p} e^{a t} q(x) \forall t \geq 0, x \in X .
$$

If $a=0$, then we simply say equicontinuous.

Given any exponentially equicontinuous $C_{0}$-semigroup $(T(t))_{t \geq 0}$ on a lcHs $X$, observe that condition (iii) in Definition 2.1 is equivalent to $T(t) \rightarrow I$ in $\mathcal{L}_{s}(X)$ as $t \rightarrow 0^{+}$.

If $X$ is a sequentially complete $\mathrm{lcHs}$ and $(T(t))_{t \geq 0}$ is a locally equicontinuous $C_{0}$-semigroup on $X$, then the linear operator $A$ defined by

$$
A x:=\lim _{t \rightarrow 0^{+}} \frac{T(t) x-x}{t},
$$

for $x \in D(A):=\left\{x \in X: \lim _{t \rightarrow 0^{+}} \frac{T(t) x-x}{t}\right.$ exists in $\left.X\right\}$, is closed with $\overline{D(A)}=X,[20$, Propositions $1.3 \& 1.4]$. The operator $(A, D(A))$ is called the infinitesimal generator of $(T(t))_{t \geq 0}$. Moreover, $A$ and $(T(t))_{t \geq 0}$ commute, $[20$, Proposition 1.2(1)], i.e., for each $t \geq 0$ we have $\{T(t) x: x \in D(A)\} \subseteq D(A)$ and $A T(t) x=T(t) A x$, for all $x \in D(A)$. Also known, [20, Proposition 1.2(2)], is that

$$
T(t) x-x=\int_{0}^{t} T(s) A x d s=\int_{0}^{t} A T(s) x d s, \quad x \in D(A),
$$

and, [20, Corollary p.261], that

$$
T(t) x-x=A \int_{0}^{t} T(s) x d s, \quad x \in X .
$$

For each $x \in D(A)$ (resp. $x \in X$ ), the integrals occurring in (2.3) (resp. (2.4)) are Riemann integrals of an $X$-valued, continuous function on $[0, t]$; see [4, Appendix]. The closedness of $A$ ensures that Ker $A:=\{x \in D(A): A x=0\}$ is a closed subspace of $X$. The range of $A$ is the subspace $\operatorname{rg} A:=$ $\{A x: x \in D(A)\}$.

Let $A: D(A) \subseteq X \rightarrow X$ be a linear operator on a lcHs $X$. If $\lambda \in \mathbb{C}$ is such that $(\lambda I-A): D(A) \rightarrow X$ is injective, the linear operator $(\lambda I-A)^{-1}$ exists and is defined on the domain $\operatorname{rg}(\lambda I-A)$. The resolvent set of $A$ is defined by

$$
\rho(A):=\left\{\lambda \in \mathbb{C}:(\lambda I-A): D(A) \rightarrow X \text { is bijective and }(\lambda I-A)^{-1} \in \mathcal{L}(X)\right\}
$$

and the spectrum of $A$ is defined by $\sigma(A):=\mathbb{C} \backslash \rho(A)$. For $\lambda \in \rho(A)$ we also write $R(\lambda, A):=(\lambda I-A)^{-1}$. For $\lambda, \mu \in \rho(A)$ it is routine to check that the resolvent equation

$$
R(\lambda, A)-R(\mu, A)=(\mu-\lambda) R(\lambda, A) R(\mu, A)
$$

is valid. The spectral theory for closed linear operators $A$ in a (non-normable) lcHs $X$ is not as well developed as in Banach spaces and many features depart from the well known theory in Banach spaces; see [5, Section 3], for example, where those aspects that we require in this paper can be found.

\section{Dissipative operators on locally convex Hausdorff spaces}

Let $A: D(A) \subseteq X \rightarrow X$ be a linear operator on a lcHs $X$ (briefly, $(A, D(A)$ ) is an operator on $X)$. Recall that the operator $(A, D(A))$ is said to be closed if for each net $\left(x_{\alpha}\right)_{\alpha} \subseteq D(A)$ satisfying $x_{\alpha} \rightarrow x$ and $A x_{\alpha} \rightarrow y$ in $X$, we have $x \in D(A)$ and $A x=y$. It is a well-known fact that if $\mathcal{G}(A):=\{(x, y) \in X \times X: x \in D(A), A x=y\}$ denotes the graph of $(A, D(A))$, the operator $(A, D(A))$ is closed if and only if its graph $\mathcal{G}(A)$ is a closed subspace of space $X \times X$ endowed with the product lc-topology. 
Definition 3.1. Let $(A, D(A))$ be an operator on a lcHs $X$. An operator $(B, D(B))$ on $X$ is called an extension of $(A, D(A))$ if $D(A) \subseteq D(B)$ and $\left.B\right|_{D(A)}=A$. The operator $(A, D(A))$ is called closable if it admits a closed extension. The smallest closed extension of a closable operator $(A, D(A))$ is called the closure of $(A, D(A))$ and it is denoted by $(\bar{A}, D(\bar{A}))$.

Remark 3.2. Let $X$ be a lcHs. A subset $\mathcal{G}$ of $X \times X$ is a graph of an operator on $X$ if and only if $\mathcal{G}$ is a subspace of $X \times X$ and $(0, y) \in \mathcal{G}$ implies $y=0$. In particular, every subspace of a graph is also a graph.

To see this fact, first assume that $\mathcal{G}$ is the graph of some operator. Then $\mathcal{G}$ clearly satisfies the property above.

Conversely, suppose that $\mathcal{G}$ satisfies the property above and set

$$
D(A):=\{x \in X: \exists y \in X \text { with }(x, y) \in \mathcal{G}\} .
$$

If $x \in D(A)$, then the corresponding $y \in X$ is unique. Indeed, if there exist $y, y^{\prime} \in X$ such that $(x, y),\left(x, y^{\prime}\right) \in \mathcal{G}$, then $\left(0, y-y^{\prime}\right) \in \mathcal{G}$ as $\mathcal{G}$ is a subspace of $X \times X$. It follows that $y=y^{\prime}$ by the hypothesis on $\mathcal{G}$. On the other hand, $D(A)$ is clearly a subspace of $X\left(x, x^{\prime} \in D(A) \Rightarrow \exists y, y^{\prime} \in X\right.$ with $(x, y),\left(x^{\prime}, y^{\prime}\right) \in \mathcal{G}$ and hence, $\left(x+x^{\prime}, y+y^{\prime}\right) \in \mathcal{G}$; this implies that $\left.x+x^{\prime} \in D(A)\right)$. Finally, if we define $A x:=y$ on $D(A)$, then $A$ is linear as it easily follows via the hypothesis on $\mathcal{G}$. Thus, $(A, D(A))$ is an operator with $\mathcal{G}(A)=\mathcal{G}$.

Proposition 3.3. Let $(A, D(A))$ be an operator on a lcHs $X$. If $(A, D(A))$ is closable, then $\mathcal{G}(\bar{A})=$ $\overline{\mathcal{G}(A)}$.

Proof. If $(B, D(B))$ is any closed extension of $(A, D(A))$, then $\mathcal{G}(A) \subseteq \mathcal{G}(B)$ and hence, $\overline{\mathcal{G}(A)} \subseteq$ $\overline{\mathcal{G}(B)}=\mathcal{G}(B)$. So, by Remark 3.2 the space $\overline{\mathcal{G}(A)}$ is the graph of some operator $(R, D(R))$ on $X$. We observe that $(B, D(B))$ is also a closed extension of $(R, D(R))$ and that $(R, D(R))$ is a closed operator on $X$ as its graph is closed. On the other hand, $(R, D(R))$ is a closed extension of $(A, D(A))$ as $\mathcal{G}(A) \subset \overline{\mathcal{G}(A)}=\mathcal{G}(R)$. Since $(B, D(B))$ is arbitrary and $(\bar{A}, D(\bar{A}))$ is the smallest closed extension of $A$, it follows that $(\bar{A}, D(\bar{A}))=(R, D(R))$ and hence, that $\mathcal{G}(\bar{A})=\overline{\mathcal{G}(A)}$.

Proposition 3.4. Let $(A, D(A))$ be an operator on a lcHs $X$. Then:

(i) $(A, D(A))$ is closable if and only if for every net $\left(x_{\alpha}\right)_{\alpha} \subseteq D(A)$ such that $x_{\alpha} \rightarrow 0$ and $A x_{\alpha} \rightarrow y$ in $X$, we have $y=0$.

(ii) If $(A, D(A))$ is closable, then $D(\bar{A})=\left\{x \in X: \exists\left(x_{\alpha}\right)_{\alpha} \subseteq D(A)\right.$ such that $x_{\alpha} \rightarrow x$ and $A x_{\alpha} \rightarrow$ $y$ in $X\}$. Moreover, $\bar{A} x=\lim _{\alpha} A x_{\alpha}$ for $x \in D(\bar{A})$.

Proof. (i) Suppose that $(A, D(A))$ is closable and that $(B, D(B))$ is any closed extension of $(A, D(A))$. Let $\left(x_{\alpha}\right)_{\alpha} \subseteq D(A)$ satisfy $x_{\alpha} \rightarrow 0$ and $A x_{\alpha} \rightarrow y$ in $X$. Then $(0, y) \in \mathcal{G}(B)$ and hence, $y=0$; see Remark 3.2.

Conversely, consider $\overline{\mathcal{G}(A)}$, which is a closed subspace of $X \times X$, being it the closure of a subspace of $X \times X$. If $(0, y) \in \overline{\mathcal{G}(A)}$, then $y=0$. Indeed, if $(0, y) \in \overline{\mathcal{G}(A)}$, then there exists $\left(x_{\alpha}\right)_{\alpha} \subseteq D(A)$ such that $x_{\alpha} \rightarrow 0$ and $A x_{\alpha} \rightarrow y$ in $X$. So, by hypothesis, $y=0$. Therefore, $\overline{\mathcal{G}(A)}$ is the graph of some closed operator (closed because the graph is closed) which is clearly a closed extension of $(A, D(A))$.

(ii) If $(A, D(A))$ is closable, then $\mathcal{G}(\bar{A})=\overline{\mathcal{G}(A)}$; see Proposition 3.3. Hence,

$$
D(\bar{A})=\{x \in X: \exists y \in X \text { with }(x, y) \in \mathcal{G}(\bar{A})=\overline{\mathcal{G}(A)}\}
$$

and $\bar{A} x=y$, where $y$ is the unique element of $X$ such that $(x, y) \in \mathcal{G}(\bar{A})=\overline{\mathcal{G}(A)}$. Consequently, if $x \in D(\bar{A})$, then $(x, y) \in \overline{\mathcal{G}(A)}$ and so there exists $\left(\left(x_{\alpha}, A x_{\alpha}\right)\right)_{\alpha} \subseteq \mathcal{G}(A)$ such that $x_{\alpha} \rightarrow x$ and $A x_{\alpha} \rightarrow y$ in $X$. Therefore, $x \in D:=\left\{z \in X: \exists\left(z_{\alpha}\right)_{\alpha} \subseteq D(A)\right.$ such that $z_{\alpha} \rightarrow z$ and $A z_{\alpha} \rightarrow w$ in $\left.X\right\}$ and $\bar{A} x=\lim _{\alpha} A_{\alpha} x$.

Conversely, if $x \in D$, where $D$ is defined as above, there exists $\left(x_{\alpha}\right)_{\alpha} \subseteq D(A)$ with $x_{\alpha} \rightarrow x$ and $A x_{\alpha} \rightarrow y$ in $X$. Then $\left(\left(x_{\alpha}, A x_{\alpha}\right)\right)_{\alpha} \subset \mathcal{G}(A)$ and $\left(x_{\alpha}, A x_{\alpha}\right) \rightarrow(x, y)$ in $X \times X$, thereby implying that $(x, y) \in \overline{\mathcal{G}(A)}=\mathcal{G}(\bar{A})$. Thus, $x \in D(\bar{A})$ and $\bar{A} x=y$. 
Definition 3.5. Given a densely defined operator $(A, D(A))$ on a lcHs $X$, we define the dual operator $\left(A^{\prime}, D\left(A^{\prime}\right)\right)$ of $(A, D(A))$ on $X^{\prime}$ by setting

$$
D\left(A^{\prime}\right):=\left\{x^{\prime} \in X^{\prime}: \exists y^{\prime} \in X^{\prime} \text { such that }\left\langle A x, x^{\prime}\right\rangle=\left\langle x, y^{\prime}\right\rangle, \forall x \in D(A)\right\},
$$

and $A^{\prime} x^{\prime}:=y^{\prime}$ for $x^{\prime} \in D\left(A^{\prime}\right)$.

We observe that the dual operator $\left(A^{\prime}, D\left(A^{\prime}\right)\right)$ is closed with respect to $\sigma\left(X^{\prime}, X\right)$ and hence, it is closed also with respect to $\beta\left(X^{\prime}, X\right)$. Indeed, if $\left(x_{\alpha}^{\prime}\right)_{\alpha} \subseteq D\left(A^{\prime}\right)$ with $x_{\alpha}^{\prime} \rightarrow x^{\prime}$ and $A x_{\alpha}^{\prime} \rightarrow y^{\prime}$ in $X_{\sigma}^{\prime}$, then $\left\langle A x, x_{\alpha}^{\prime}\right\rangle=\left\langle x, A^{\prime} x_{\alpha}^{\prime}\right\rangle$ for all $x \in D(A)$ and $\alpha$. Passing to the limit on $\alpha$, we get that

$$
\left\langle A x, x^{\prime}\right\rangle=\left\langle x, y^{\prime}\right\rangle, \quad x \in D(A) .
$$

This yields that $x^{\prime} \in D\left(A^{\prime}\right)$ and $A^{\prime} x^{\prime}=y^{\prime}$.

Now, we collect some properties of dual operators.

Proposition 3.6. Let $(A, D(A))$ be a densely defined operator on a lcHs $X$. Then:

(i) $\operatorname{ker} A^{\prime}=(\operatorname{rg} A)^{\perp}$.

(ii) If $D\left(A^{\prime}\right)$ is dense in $X_{\beta}^{\prime}$, then the dual operator $\left(A^{\prime \prime}, D\left(A^{\prime \prime}\right)\right)$ of $\left(A^{\prime}, D\left(A^{\prime}\right)\right)$ is an extension of $(A, D(A))$.

(iii) If $A: D(A) \subseteq X \rightarrow X$ is a continuous linear operator, then $A^{\prime}: D\left(A^{\prime}\right) \subseteq X_{\beta}^{\prime} \rightarrow X_{\beta}^{\prime}$ is a continuous linear operator and $D\left(A^{\prime}\right)=X^{\prime}$. The converse holds if, in addition, $X$ is barrelled.

Proof. (i) Observe that $x^{\prime} \in(\operatorname{rg} A)^{\perp}$ if and only if $\left\langle x, x^{\prime}\right\rangle=0$ for all $x \in \operatorname{rg} A=\{A z: z \in D(A)\}$, i.e., $\left\langle A z, x^{\prime}\right\rangle=0$ for all $z \in D(A)$. Since $\langle z, 0\rangle=0$ for all $z \in D(A)$, this is equivalent to the fact that $x^{\prime} \in D\left(A^{\prime}\right)$ and $A^{\prime} x^{\prime}=0$, i.e., $x^{\prime} \in \operatorname{ker} A^{\prime}=\left\{y^{\prime} \in D(A): A^{\prime} y^{\prime}=0\right\}$.

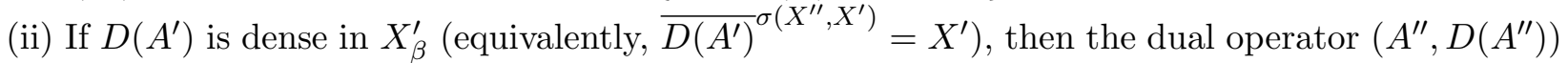
of $\left(A^{\prime}, D\left(A^{\prime}\right)\right)$ is well defined on $X^{\prime \prime}$. Moreover, if $x \in D(A)$, then $\left\langle x, A^{\prime} x^{\prime}\right\rangle=\left\langle A x, x^{\prime}\right\rangle$ for all $x^{\prime} \in D\left(A^{\prime}\right)$, where $x$ and $A x$ belong to $X$ and hence, also to $X^{\prime \prime}$ as $X \subseteq X^{\prime \prime}$. Thus, the equality above ensures that $x \in D\left(A^{\prime \prime}\right)$ and $A^{\prime \prime} x=A x$.

(iii) Since $A$ is continuous, $A$ admits a unique continuous linear extension $\bar{A}$ on $X$, i.e., $\bar{A} \in \mathcal{L}(X)$. So, $\bar{A}^{\prime} \in \mathcal{L}\left(X_{\beta}^{\prime}\right)$ and $\left\langle\bar{A} x, x^{\prime}\right\rangle=\left\langle x, \bar{A}^{\prime} x^{\prime}\right\rangle$ for all $x \in X$ and $x^{\prime} \in X^{\prime}$. Since $\left.\bar{A}\right|_{D(A)}=A$, it follows, for every $x \in D(A)$ and $x^{\prime} \in X^{\prime}$, that

$$
\left\langle A x, x^{\prime}\right\rangle=\left\langle x, \bar{A}^{\prime} x^{\prime}\right\rangle .
$$

This proves that $D\left(A^{\prime}\right)=X^{\prime}$ and that $A^{\prime}=\bar{A}^{\prime} \in \mathcal{L}\left(X_{\beta}^{\prime}\right)$.

Suppose that $A^{\prime} \in \mathcal{L}\left(X_{\beta}^{\prime}\right)$. Then $A^{\prime \prime} \in \mathcal{L}\left(X_{\beta}^{\prime \prime}\right)$ and hence, also $A^{\prime \prime} \in \mathcal{L}\left(\left(X^{\prime \prime}, \sigma\left(X^{\prime \prime}, X^{\prime}\right)\right)\right)$, where by part (ii) of this proposition the operator $\left(A^{\prime \prime}, D\left(A^{\prime \prime}\right)\right)$ is an extension of $(A, D(A))$, i.e., for every $x \in D(A)$, we have $A x=A^{\prime \prime} x$. If, in addition, $X$ is barrelled, the lc-topology induced by $X_{\beta}^{\prime \prime}$ on $X$ coincides with its original lc-topology. Then it follows that $A=\left.A^{\prime \prime}\right|_{D(A)}$ is also continuous on $X$.

Moreover, we have

Proposition 3.7. Let $(A, D(A))$ be a densely defined operator on a lcHs $X$. Then:

(i) If $(A, D(A))$ is closable, then $\bar{A}^{\prime}=A^{\prime}$.

(ii) Let $X$ be barrelled. If $D\left(A^{\prime}\right)$ is dense in $X_{\beta}^{\prime}$, then $(A, D(A))$ is closable. The converse holds if, in addition, $X$ is reflexive. In such a case, we have $\bar{A}=\left.A^{\prime \prime}\right|_{D(\bar{A})}$ with $D(\bar{A})=\{x \in$ $\left.D\left(A^{\prime \prime}\right) \cap X: A^{\prime \prime} x \in X\right\}$.

Proof. (i) Fix $x^{\prime} \in D\left(\bar{A}^{\prime}\right)$. Then $\left\langle\bar{A} x, x^{\prime}\right\rangle=\left\langle x, \bar{A}^{\prime} x^{\prime}\right\rangle$ for all $x \in D(\bar{A})$. Since $D(A) \subseteq D(\bar{A})$ and $\left.\bar{A}\right|_{D(A)}=A$, it follows that $\left\langle A x, x^{\prime}\right\rangle=\left\langle x, \bar{A}^{\prime} x^{\prime}\right\rangle$ for all $x \in D(A)$ and hence, $x^{\prime} \in D\left(A^{\prime}\right)$ and $A^{\prime} x^{\prime}=\bar{A}^{\prime} x^{\prime}$.

Conversely, if $x^{\prime} \in D\left(A^{\prime}\right)$, then $\left\langle A x, x^{\prime}\right\rangle=\left\langle x, A^{\prime} x^{\prime}\right\rangle$ for all $x \in D(A)$. This equality extends to the whole space $D(\bar{A})$. Indeed, for $x \in D(\bar{A})$ fixed, by Proposition 3.4(i) there exists a net $\left(x_{\alpha}\right)_{\alpha} \subseteq D(A)$ such that $x_{\alpha} \rightarrow x$ and $A x_{\alpha} \rightarrow \bar{A} x$ in $X$. Since $\left\langle A x_{\alpha}, x^{\prime}\right\rangle=\left\langle x_{\alpha}, A^{\prime} x^{\prime}\right\rangle$ for all $\alpha$, by passing to the limit on $\alpha$ it follows that $\left\langle\bar{A} x, x^{\prime}\right\rangle=\left\langle x, A^{\prime} x^{\prime}\right\rangle$. The arbitrariness of $x$ in $D(\bar{A})$ yields that $x^{\prime} \in D\left(\bar{A}^{\prime}\right)$ and $\bar{A}^{\prime} x^{\prime}=A^{\prime} x^{\prime}$. 
(ii) If $D\left(A^{\prime}\right)$ is dense in $X_{\beta}^{\prime}$, then its dual operator $\left(A^{\prime \prime}, D\left(A^{\prime \prime}\right)\right)$ is well defined and closed in $X_{\beta}^{\prime \prime}$; see the comment below Definition 3.5. Moreover, by Proposition 3.6(ii) the operator $\left(A^{\prime \prime}, D\left(A^{\prime \prime}\right)\right)$ is an extension of $(A, D(A))$. Since $X$ is barrelled, it follows that $(A, D(A))$ is closable. Indeed, if $\left(x_{\alpha}\right)_{\alpha} \subseteq D(A) \subseteq X$ such that $x_{\alpha} \rightarrow 0$ and $A x_{\alpha} \rightarrow y$ in $X$, then $\left(x_{\alpha}\right)_{\alpha} \subseteq D\left(A^{\prime \prime}\right)$ also satisfies the conditions $x_{\alpha} \rightarrow 0$ and $A^{\prime \prime} x_{\alpha}=A x_{\alpha} \rightarrow y$ in $X_{\beta}^{\prime \prime}$. The closedness of $\left(A^{\prime \prime}, D\left(A^{\prime \prime}\right)\right)$ implies that $y=0$. So, by Proposition 3.4(i) we conclude that $(A, D(A))$ is closable.

Suppose that $X$ is reflexive and that $D\left(A^{\prime}\right)$ is not dense in $X_{\beta}^{\prime}$ (and hence, $D\left(A^{\prime}\right)$ is not dense in $\left.\left(X^{\prime}, \sigma\left(X^{\prime}, X\right)\right)\right)$. Then there exists $x \in X$ such that $x \neq 0$ and $\left\langle x, x^{\prime}\right\rangle=0$ for all $x^{\prime} \in D\left(A^{\prime}\right)$, i.e., $x \in\left(D\left(A^{\prime}\right)\right)^{\perp}$. This implies that $(0, x) \in \overline{\mathcal{G}(A)}$. Indeed, if $(0, x) \notin \overline{\mathcal{G}(A)}$, then by Hahn-Banach theorem there exists $\left(y^{\prime}, z^{\prime}\right) \in X^{\prime} \times X^{\prime}$ such that $1=\left\langle(0, x),\left(y^{\prime}, z^{\prime}\right)\right\rangle=\left\langle 0, y^{\prime}\right\rangle+\left\langle x, z^{\prime}\right\rangle=\left\langle x, z^{\prime}\right\rangle$ and

$$
0=\left\langle(w, A w),\left(y^{\prime}, z^{\prime}\right)\right\rangle=\left\langle w, y^{\prime}\right\rangle+\left\langle A w, z^{\prime}\right\rangle
$$

for all $w \in D(A)$. Hence, $\left\langle A w, z^{\prime}\right\rangle=\left\langle w,-y^{\prime}\right\rangle$ for all $w \in D(A)$ with $z^{\prime}, y^{\prime} \in X^{\prime}$. This means that $z^{\prime} \in D\left(A^{\prime}\right)$ and $A^{\prime} z^{\prime}=-y^{\prime}$. Then, $\left\langle x, z^{\prime}\right\rangle=0$; this is a contradiction with the fact that $\left\langle x, z^{\prime}\right\rangle=1$.

The fact that $(0, x) \in \overline{\mathcal{G}(A)}$ with $x \neq 0$ means that $\overline{\mathcal{G}(A)}$ is not the graph of an operator (see Remark 3.2) and hence, that $(A, D(A))$ is not closable. Moreover, when $X$ is reflexive, we have $\bar{A}=\left.A^{\prime \prime}\right|_{D(\bar{A})}$ with $D(\bar{A})=\left\{x \in D\left(A^{\prime \prime}\right) \cap X: A^{\prime \prime} x \in X\right\}$.

Example 3.8. Let $X=C(\mathbb{R})$ be the Fréchet space of continuous functions on $\mathbb{R}$ endowed with the compact-open topology (i.e., its lc-topology is generated by the seminorms $p_{h}(f):=\sup _{x \in[-h, h]}|f(x)|$, $h \in \mathbb{N}$ and $f \in X$, and so $X$ is a non-normable Fréchet space) and consider the operator

$$
A f:=f^{\prime}(0) \cdot \mathbb{1}, \quad f \in D(A)=C^{1}(\mathbb{R}) .
$$

Then, $\left(A, C^{1}(\mathbb{R})\right)$ is not closable. In fact, if $f_{n}(x)=\frac{1}{n} \sin (n x+\pi)$, for $n \in \mathbb{N}$ and $x \in \mathbb{R}$, then $f_{n} \rightarrow 0$ uniformly in $\mathbb{R}$ and so $f_{n} \rightarrow 0$ in $X$. On the other hand, $f_{n}^{\prime}(x)=\cos (n x+\pi)$, for $n \in \mathbb{N}$ and $x \in \mathbb{R}$, and hence, $A f_{n}=-\mathbb{1}$ which tends to $-\mathbb{1}$ in $X$. Since $-\mathbb{1} \neq 0$, by Proposition 3.4(i) it follows that $\left(A, C^{1}(\mathbb{R})\right)$ is not closable.

Since $D(A)=C^{1}(\mathbb{R})$ is dense in $X$, there exists the dual operator $\left(A^{\prime}, D\left(A^{\prime}\right)\right)$ of $\left(A, C^{1}(\mathbb{R})\right)$. To determine the dual operator $\left(A^{\prime}, D\left(A^{\prime}\right)\right)$ we proceed as follows.

Let $F \in D\left(A^{\prime}\right)$. Then $\langle A f, F\rangle=\left\langle f, A^{\prime} F\right\rangle$, for all $f \in D(A)$, i.e., $f^{\prime}(0) \cdot F(\mathbb{1})=\left\langle f^{\prime}(0) \cdot \mathbb{1}, F\right\rangle=$ $\left\langle f, A^{\prime} F\right\rangle$, for all $f \in D(A)$. If $F(\mathbb{1}) \neq 0$, then $f^{\prime}(0)=\frac{1}{F(\mathbb{1})}\left(A^{\prime} F\right)(f)$ for all $f \in D(A)$ and hence, as $A^{\prime} F \in X^{\prime}$, there exist $M>0$ and $h \in \mathbb{N}$ such that

$$
\left|f^{\prime}(0)\right| \leq \frac{1}{|F(\mathbb{1})|} M p_{h}(f), \quad f \in D(A) .
$$

Since $f_{n}(x)=\frac{1}{n} \sin (n x+\pi) \in D(A)$ for all $n \in \mathbb{N}$, it follows that

$$
1=\left|f_{n}^{\prime}(0)\right| \leq \frac{1}{|F(\mathbb{1})|} M p_{h}\left(f_{n}\right) \leq \frac{1}{|F(\mathbb{1})|} \frac{M}{n}, \quad n \in \mathbb{N},
$$

which is a contradiction for large $n \in \mathbb{N}$. Then, $F(\mathbb{1})=0$. So, the fact that $f^{\prime}(0) \cdot F(\mathbb{1})=\left\langle f, A^{\prime} F\right\rangle$ for all $f \in D(A)$ implies that $\left\langle f, A^{\prime} F\right\rangle=0$ for all $f \in D(A)$ and hence, that $A^{\prime} F=0$ in $X$, being $D(A)$ dense in $X$. Since $F \in D\left(A^{\prime}\right)$ is arbitrary, we can conclude that $A^{\prime}=0$ on $D\left(A^{\prime}\right)=\left\{F \in X^{\prime}: F(\mathbb{1})=0\right\}$. Moreover, the fact that $(A, D(A))$ is not closable implies via Proposition 3.7(i) that $D\left(A^{\prime}\right)$ cannot be dense in $X_{\beta}^{\prime}$.

Definition 3.9. Let $X$ be a lcHs. An operator $(A, D(A))$ is called dissipative if there exists $\Gamma \subseteq \Gamma_{X}$ determining the lc-topology of $X$ such that

$$
p((\lambda I-A) x) \geq \lambda p(x), \quad \lambda>0, x \in D(A), p \in \Gamma .
$$

In such a case, we also say that $(A, D(A))$ is $\Gamma$-dissipative.

Proposition 3.10. Let $X$ be a lcHs and $(A, D(A))$ be a $\Gamma$-dissipative operator on $X$. Then: 
(i) $\lambda I-A$ is injective for every $\lambda>0$. Moreover, we have

$$
p\left((\lambda I-A)^{-1} z\right) \leq \frac{1}{\lambda} p(z), \quad \lambda>0, z \in \operatorname{rg}(\lambda I-A), p \in \Gamma .
$$

(ii) If $\operatorname{rg}(\lambda I-A)$ is (sequentially) closed for some $\lambda>0$, then $(A, D(A))$ is (sequentially) closed. If, in addition, $X$ is (sequentially) complete, then the converse holds, even for all $\lambda>0$.

(iii) Let $X$ be sequentially complete. Then $\lambda I-A$ is onto for some $\lambda>0$ if and only if it is onto for all $\lambda>0$. In such a case, $(0,+\infty) \subset \rho(A)$.

Proof. (i) Fix $\lambda>0$ and suppose that $(\lambda I-A) x=0$ for some $x \in D(A)$. Then, from (3.1) it follows that

$$
0=p((\lambda I-A) x) \geq \lambda p(x) \geq 0, \quad p \in \Gamma,
$$

i.e., $p(x)=0$ for all $p \in \Gamma$ and hence, $x=0$. As $x$ is arbitrary, it follows that $\lambda I-A$ is injective.

Since $\lambda I-A$ is injective, the inequalities in (3.2) easily follow from (3.1).

(ii) Suppose that $\operatorname{rg}(\lambda I-A)$ is closed for some $\lambda>0$. To prove that $(A, D(A))$ is closed, fix a net $\left(x_{\alpha}\right)_{\alpha} \subseteq D(A)$ satisfying $x_{\alpha} \rightarrow x$ and $A x_{\alpha} \rightarrow y$ in $X$. Then $(\lambda I-A) x_{\alpha}=\lambda x_{\alpha}-A x_{\alpha} \rightarrow \lambda x-y$ in $X$, where $\left((\lambda I-A) x_{\alpha}\right)_{\alpha} \subseteq \operatorname{rg}(\lambda I-A)$. Since $\operatorname{rg}(\lambda I-A)$ is closed, it follows that $\lambda x-y \in \operatorname{rg}(\lambda I-A)$, i.e., $\lambda x-y=(\lambda I-A) z$ for some $z \in D(A)$.

Now, we observe that by part (i) of this proposition the operator $(\lambda I-A)^{-1}: \operatorname{rg}(\lambda I-A) \subseteq X \rightarrow X$ exists, is continuous and satisfies $\operatorname{rg}(\lambda I-A)^{-1}=D(A)$. As $(\lambda I-A) x_{\alpha} \rightarrow \lambda x-y$ in $\operatorname{rg}(\lambda I-A)$, we have $x_{\alpha}=(\lambda I-A)^{-1}(\lambda I-A) x_{\alpha} \rightarrow(\lambda I-A)^{-1}(\lambda x-y)$. But, $x_{\alpha} \rightarrow x$ in $X$. Thus, $(\lambda I-A)^{-1}(\lambda x-y)=x$. Since $\operatorname{rg}(\lambda I-A)^{-1}=D(A)$, this implies that $x \in D(A)$ and hence, we also have $(\lambda I-A)^{-1}(\lambda I-A) x=$ $x$. By the injectivity of $(\lambda I-A)^{-1}$, it follows that $\lambda x-y=\lambda x-A x$ and so $y=A x$. Therefore, $(A, D(A))$ is closed.

Conversely, suppose that $X$ is complete and that $(A, D(A))$ is closed. Fixed any $\lambda>0$, let $\left(y_{\alpha}\right)_{\alpha} \subseteq$ $\operatorname{rg}(\lambda I-A)$ satisfying $y_{\alpha} \rightarrow y$ in $X$. Since $y_{\alpha}=(\lambda I-A) x_{\alpha}$ with $x_{\alpha} \in D(A)$ for all $\alpha$, from (3.1) it follows that

$$
p\left(x_{\alpha}-x_{\alpha}^{\prime}\right) \leq \frac{1}{\lambda} p\left(y_{\alpha}-y_{\alpha}^{\prime}\right),
$$

for all $p \in \Gamma$ and $\alpha, \alpha^{\prime}$. Since $\left(y_{\alpha}\right)_{\alpha}$ is convergent in $X$, it then follows that $\left(x_{\alpha}\right)_{\alpha}$ is a Cauchy net in $X$ and hence, that $x_{\alpha} \rightarrow x$ in $X$, being $X$ complete. Therefore, $\left(x_{\alpha}\right)_{\alpha} \subseteq D(A)$ is a net satisfying $x_{\alpha} \rightarrow x$ and $(\lambda I-A) x_{\alpha} \rightarrow y$ in $X$. Since $(A, D(A))$ is closed, we obtain $x \in D(A)$ and $y=(\lambda I-A) x$ and so $y \in \operatorname{rg}(\lambda I-A)$.

Proceeding in a similar way, one shows that if $\operatorname{rg}(\lambda I-A)$ is sequentially closed for some $\lambda>0$, then $(A, D(A))$ is sequentially closed and that the converse holds when $X$ is, in addition, sequentially complete.

(iii) It suffices to show only the necessary condition. So, suppose that $\left(\lambda_{0} I-A\right): D(A) \subseteq X \rightarrow X$ is surjective for some $\lambda_{0}>0$. Then, by part (i) of this proposition we have that

$$
R\left(\lambda_{0}\right):=\left(\lambda_{0} I-A\right)^{-1}: X \rightarrow X,
$$

with $\operatorname{rg} R\left(\lambda_{0}\right)=D(A)$, is a continuous linear operator on $X$ satisfying

$$
p\left(R\left(\lambda_{0}\right) x\right) \leq \frac{1}{\lambda_{0}} p(x), \quad x \in X, p \in \Gamma .
$$

From (3.3) it clearly follows that

$$
p\left(\left[R\left(\lambda_{0}\right)\right]^{n} x\right) \leq \frac{1}{\left(\lambda_{0}\right)^{n}} p(x), \quad x \in X, p \in \Gamma, n \in \mathbb{N} .
$$

This yields that the series

$$
\sum_{n=0}^{\infty}\left(\lambda_{0}-\mu\right)^{n}\left[R\left(\lambda_{0}\right)\right]^{n+1}
$$


converges in $\mathcal{L}_{b}(X)$, say to $R(\mu)$, for all $\mu \in\left(0,2 \lambda_{0}\right)$. Indeed, by (3.4) we obtain, for every $x \in X$, $n \in \mathbb{N}, p \in \Gamma$ and $\mu>0$, that

$$
p\left(\left(\lambda_{0}-\mu\right)^{n}\left[R\left(\lambda_{0}\right)\right]^{n+1} x\right) \leq \frac{1}{\lambda_{0}}\left(\frac{\left|\lambda_{0}-\mu\right|}{\lambda_{0}}\right)^{n} p(x)
$$

and hence, for every $B \in \mathcal{B}(X)$, that

$$
\sup _{x \in B} p\left(\left(\lambda_{0}-\mu\right)^{n}\left[R\left(\lambda_{0}\right)\right]^{n} x\right) \leq \frac{1}{\lambda_{0}}\left(\frac{\left|\lambda_{0}-\mu\right|}{\lambda_{0}}\right)^{n} \sup _{x \in B} p(x) .
$$

Fixed $p \in \Gamma$ and $B \in \mathcal{B}(X)$ and observing that $M:=\sup _{x \in B} p(x)<\infty$, it follows that the series $\sum_{n=0}^{\infty} \sup _{x \in B} p\left(\left(\lambda_{0}-\mu\right)^{n}\left[R\left(\lambda_{0}\right)\right]^{n} x\right)$ surely converges if $\frac{\left|\lambda_{0}-\mu\right|}{\lambda_{0}}<1$, i.e., if $\mu \in\left(0,2 \lambda_{0}\right)$.

Moreover, for every $\mu \in\left(0,2 \lambda_{0}\right)$, the operator $(\mu-A): D(A) \rightarrow X$ is surjective (hence, bijective by part (i) of this proposition) with $(\mu-A)^{-1}=R(\mu)$. In fact,

$$
\begin{aligned}
R(\mu)(\mu I-A) & =\sum_{n=0}^{\infty}\left(\lambda_{0}-\mu\right)^{n}\left[R\left(\lambda_{0}\right)\right]^{n+1}(\mu I-A) \\
& =\sum_{n=0}^{\infty}\left(\lambda_{0}-\mu\right)^{n}\left[R\left(\lambda_{0}\right)\right]^{n+1}\left[\left(\lambda_{0} I-A\right)-\left(\mu-\lambda_{0}\right) I\right] \\
& =\sum_{n=0}^{\infty}\left[\left(\lambda_{0}-\mu\right)^{n}\left(R\left(\lambda_{0}\right)\right)^{n}-\left(\lambda_{0}-\mu\right)^{n+1}\left(R\left(\lambda_{0}\right)\right)^{n+1}\right] \\
& =\lim _{k \rightarrow \infty} \sum_{n=0}^{k}\left[\left(\lambda_{0}-\mu\right)^{n}\left(R\left(\lambda_{0}\right)\right)^{n}-\left(\lambda_{0}-\mu\right)^{n+1}\left(R\left(\lambda_{0}\right)\right)^{n+1}\right] \\
& =\lim _{k \rightarrow \infty}\left[I-\left(\lambda_{0}-\mu\right)^{n+1}\left(R\left(\lambda_{0}\right)\right)^{n+1}\right]=I .
\end{aligned}
$$

In a similar way, one shows that $(\mu I-A) R(\mu)=I$. Therefore, the operator $\mu I-A$ is surjective with $(\mu I-A)^{-1}=R(\mu)$.

Iterating such a procedure with $2 \lambda_{0}$ instead of $\lambda_{0}$ and so on, the result follows.

Remark 3.11. If $(A, D(A))$ is the infinitesimal generator of an equicontinuous $C_{0}$-semigroup $(T(t))_{t \geq 0}$ on a sequentially complete lcHs $X$, then $(A, D(A))$ is a dissipative, closed densely defined operator on $X$.

In fact, in such a case $(A, D(A))$ is a closed densely defined operator on $X$ such that $\mathbb{C}_{+} \subseteq \rho(A)$ and

$$
R(\lambda, A) x=\int_{0}^{+\infty} e^{-\lambda t} T(t) x d t, \quad \lambda \in \mathbb{C}_{+}, x \in X
$$

see [20, Proposition 1.4] and [28, Corollary 1, p.241]. Moreover, by the equicontinuity of $(T(t))_{t \geq 0}$ we may assume that

$$
p(T(t) x) \leq p(x), \quad x \in X, t \geq 0, p \in \Gamma_{X} ;
$$

see [5, Remark 2.2(i)]. So, it follows via (3.5) that,

$$
p(R(\lambda, A) x) \leq \frac{1}{\operatorname{Re} \lambda} p(x), \quad \lambda \in \mathbb{C}_{+}, x \in X, p \in \Gamma_{X} ;
$$

see [5, Remark 3.5(iv)]. In particular, by (3.6) we get

$$
p(x)=p(R(\lambda, A)(\lambda I-A) x) \leq \frac{1}{\lambda} p((\lambda I-A) x), \quad \lambda>0, x \in D(A), p \in \Gamma_{X} .
$$

Accordingly, $(A, D(A))$ is dissipative.

Proposition 3.12. Let $(A, D(A))$ be a $\Gamma$-dissipative operator on a lcHs $X$. If $\overline{D(A)}=X$, then $(A, D(A))$ is closable and its closure $(\bar{A}, D(\bar{A}))$ is also a dissipative operator on $X$. Moreover, if $X$ is complete, then $\operatorname{rg}(\lambda I-\bar{A})=\overline{\operatorname{rg}(\lambda I-A)}$ for all $\lambda>0$. 
Proof. By Proposition 3.4(i) to show that $(A, D(A))$ is closable it suffices to prove that if $\left(x_{\alpha}\right)_{\alpha} \subseteq D(A)$ satisfies $x_{\alpha} \rightarrow 0$ and $A x_{\alpha} \rightarrow y$ in $X$, then $y=0$.

So, fix a net $\left(x_{\alpha}\right)_{\alpha} \subseteq D(A)$ satisfying $x_{\alpha} \rightarrow 0$ and $A x_{\alpha} \rightarrow y$. Then by (3.1) we have

$$
p\left(\lambda(\lambda I-A) x_{\alpha}+(\lambda I-A) w\right)=p\left((\lambda I-A)\left(\lambda x_{\alpha}+w\right)\right) \geq \lambda p\left(\lambda x_{\alpha}+w\right),
$$

for all $w \in D(A), \lambda>0$ and $p \in \Gamma\left(\Gamma \subseteq \Gamma_{X}\right.$ determines the lc-topology of $\left.X\right)$. By passing to the limit with respect to $\alpha$, we obtain

$$
p(-\lambda y+(\lambda I-A) w) \geq \lambda p(w),
$$

for all $w \in D(A), \lambda>0$ and $p \in \Gamma$. Hence,

$$
p\left(-y+w-\frac{1}{\lambda} A w\right) \geq p(w),
$$

for all $w \in D(A), \lambda>0$ and $p \in \Gamma$. Letting $\lambda \rightarrow+\infty$ it follows that

$$
p(-y+w) \geq p(w)
$$

for all $w \in D(A)$ and $p \in \Gamma$, which yields that $y=0$. Indeed, if $y \neq 0$ then there is $p \in \Gamma$ such that $p(y)>0$. Let $\varepsilon:=\frac{p(y)}{2}$. Since $\overline{D(A)}=X$, there is $w \in D(A)$ such that $p(-y+w)<\varepsilon$ and so

$$
|p(y)-p(w)| \leq p(-y+w)<\varepsilon .
$$

From this we get that $p(y)-p(w)<\varepsilon=\frac{p(y)}{2}$ and hence, $\frac{1}{2} p(y)<p(w)$. Therefore, via (3.7) we have

$$
\frac{1}{2} p(y)<p(w) \leq p(-y+w)<\varepsilon=\frac{1}{2} p(y) ;
$$

this is a contradiction. So, $(A, D(A))$ is closable.

Fix $x \in D(\bar{A})$. Then, by Proposition 3.4(ii) there is $\left(x_{\alpha}\right)_{\alpha} \subseteq D(A)$ such that $x_{\alpha} \rightarrow x$ and $A x_{\alpha} \rightarrow \bar{A} x$ in $X$. On the other hand, by (3.1) we have

$$
p\left((\lambda I-A) x_{\alpha}\right) \geq \lambda p\left(x_{\alpha}\right), \quad \lambda>0, \alpha, p \in \Gamma .
$$

Passing to the limit with respect to $\alpha$, it follows thanks to the continuity of each $p \in \Gamma$ that

$$
p((\lambda I-\bar{A}) x) \geq \lambda p(x), \quad \lambda>0, p \in \Gamma .
$$

The arbitrariness of $x$ together with (3.8) imply that $(\bar{A}, D(\bar{A}))$ is dissipative.

Fixed any $\lambda>0$, we first observe that $\operatorname{rg}(\lambda I-A)=\{(\lambda I-A) x: x \in D(A)\}$ is dense in $\operatorname{rg}(\lambda I-\bar{A})$. Indeed, if $z \in \operatorname{rg}(\lambda I-\bar{A})$ then $z=(\lambda I-\bar{A}) x$ for some $x \in D(\bar{A})$. So, by Proposition 3.4(ii) there is $\left(x_{\alpha}\right)_{\alpha} \subseteq D(A)$ such that $x_{\alpha} \rightarrow x$ and $A x_{\alpha} \rightarrow \bar{A} x$ in $X$ and, hence, $\left((\lambda I-A) x_{\alpha}\right)_{\alpha} \subseteq \operatorname{rg}(\lambda I-A)$ and $(\lambda I-A) x_{\alpha} \rightarrow(\lambda I-\bar{A}) x=z$. Now, if we suppose that $X$ is also complete, then by Proposition 3.10(ii) the subspace $\operatorname{rg}(\lambda I-\bar{A})$ is closed in $X$ and so $\operatorname{rg}(\lambda I-\bar{A})=\overline{\operatorname{rg}(\lambda I-A)}$.

Now, we are able to extend in the setting of operators acting on locally convex spaces a classical result of Lumer and Phillips for unbounded operators acting on Banach spaces; see [21, Theorem 3.1].

Theorem 3.13. Let $(A, D(A))$ be a dissipative, densely defined operator on a complete lcHs $X$. Then the following statements are equivalent:

(i) The closure $(\bar{A}, D(\bar{A}))$ of $(A, D(A))$ generates an equicontinuous $C_{0}$-semigroup on $X$,

(ii) $\operatorname{rg}(\lambda I-A)$ is dense in $X$ for some $\lambda>0$ (hence, for all $\lambda>0$ ).

Proof. (i) $\Rightarrow$ (ii). The generation of an equicontinuous $C_{0}$-semigroup on $X$ implies that $\operatorname{rg}(\lambda I-\bar{A})=X$ for all $\lambda>0$; see, f.i., $[28$, Corollary 1, p. 241]. Since $\operatorname{rg}(\lambda I-\bar{A})=\overline{\operatorname{rg}(\lambda I-A)}$ by Proposition 3.12, (ii) follows.

(ii) $\Rightarrow$ (i). Since $(A, D(A))$ is a dissipative, densely defined operator on $X$ and $X$ is complete, by Proposition 3.12 the operator $(A, D(A))$ is closable with closure $(\bar{A}, D(\bar{A}))$ a dissipative operator on $X$ such that $\operatorname{rg}(\lambda I-\bar{A})=\overline{\operatorname{rg}(\lambda I-A)}$ for all $\lambda>0$. Hence, $\operatorname{rg}(\lambda I-\bar{A})$ is closed for all $\lambda>0$ (see also Proposition 3.10(ii)).

Now, let $\lambda>0$ be such that $\operatorname{rg}(\lambda I-A)$ is dense in $X$. Then $\operatorname{rg}(\lambda I-A)$ is also dense in $\operatorname{rg}(\lambda I-\bar{A})$ and so, being $\operatorname{rg}(\lambda I-\bar{A})$ a closed subspace of $X$, we have that $\operatorname{rg}(\lambda I-\bar{A})=X$, i.e., $\lambda I-\bar{A}$ is 
surjective. From Proposition 3.10(i)-(iii) it follows that the operator $(\lambda I-\bar{A}): D(\bar{A}) \subseteq X \rightarrow X$ is bijective for all $\lambda>0$ (hence, $(0,+\infty) \subset \rho(\bar{A}))$ and that

$$
p(R(\lambda, \bar{A}) x)=p\left((\lambda I-\bar{A})^{-1} x\right) \leq \frac{1}{\lambda} p(x), \quad \lambda>0, x \in X, p \in \Gamma,
$$

with $\Gamma \subseteq \Gamma_{X}$ determining the lc-topology of $X$.

By the inequalities in (3.9) we get that the set $\left\{[\lambda R(\lambda, \bar{A})]^{n}: \lambda>0, n \in \mathbb{N}\right\}$ is equicontinuous in $\mathcal{L}(X)$. Indeed, if we fix $p \in \Gamma$, by (3.9) we obtain

$$
p\left([\lambda R(\lambda, \bar{A})]^{n} x\right) \leq p(x), \quad \lambda>0, x \in X, n \in \mathbb{N} .
$$

Since $(\bar{A}, D(\bar{A}))$ is (clearly) a densely defined operator on the complete lcHs $X$ such that $(0,+\infty) \subset$ $\rho(\bar{A})$, via $(3.10)$ we can conclude that $(\bar{A}, D(\bar{A}))$ generates an equicontinuous $C_{0}$-semigroup on $X$; see [28, Theorem, p.246].

Example 3.14. Let $\mathbb{R}_{+}=\left[0,+\infty\left[\right.\right.$ and $X=C_{0}\left(\mathbb{R}_{+}\right)=\left\{f: \mathbb{R}_{+} \rightarrow \mathbb{R}: f\right.$ continuous on $\left.\mathbb{R}_{+}, f(0)=0\right\}$. Then $X$ is a Fréchet space with respect to the lc-topology generated by the seminorms $p_{h}(f):=$ $\sup _{x \in[0, h]}|f(x)|, h \in \mathbb{N}$ and $f \in X$.

We consider the operator $A f:=-f^{\prime}$, with $u \in D(A):=\left\{g \in C^{1}\left(\mathbb{R}_{+}\right): g(0)=0\right\}$. Then $(A, D(A))$ is dissipative. In fact, fixed any $\lambda>0$ and $g \in C_{0}\left(\mathbb{R}_{+}\right)$, the equation $\lambda f-A f=g$ has a solution $f \in D(A)$ given by

$$
f(x)=\int_{0}^{x} e^{\lambda(\xi-x)} g(\xi) d \xi=e^{-\lambda x} \int_{0}^{x} e^{\lambda \xi} g(\xi) d \xi .
$$

This shows that $\operatorname{rg}(\lambda I-A)=X$. Moreover, we have, for every $h \geq 1$ and $x \in[0, h]$, that

$$
\begin{aligned}
\lambda|f(x)| & \leq \lambda e^{-\lambda x} \int_{0}^{x} e^{\lambda \xi}|g(\xi)| d \xi \leq \lambda e^{-\lambda x}\left[\frac{e^{\lambda \xi}}{\lambda}\right]_{0}^{x} p_{h}(g) \\
& =e^{-\lambda x}\left(e^{\lambda x}-1\right) p_{h}(g) \leq\left(1-e^{-\lambda x}\right) p_{h}(g) \leq p_{h}(g),
\end{aligned}
$$

and hence, that $\lambda p_{h}(f) \leq p_{h}(g)=p_{h}(\lambda f-A f)$.

Since $\lambda>0$ and $g$ are arbitrary, we can conclude that $(A, D(A))$ is dissipative in $X$. Moreover, $\overline{D(A)}=X$. Accordingly, by Theorem 3.13 the operator $(A, D(A))$ generates an equicontinuous $C_{0^{-}}$ semigroup on $X$.

The same operator on $C\left(\mathbb{R}_{+}\right)$is dissipative with $\operatorname{rg}(\lambda I-A)=C\left(\mathbb{R}_{+}\right)$, for $\lambda>0$, (for the proof it suffices to proceed as above), but $\overline{D(A)}=C_{0}\left(\mathbb{R}_{+}\right) \varsubsetneqq C\left(\mathbb{R}_{+}\right)$and hence, the operator $(A, D(A))$ cannot generate an equicontinuous $C_{0}$-semigroup on $C\left(\mathbb{R}_{+}\right)$.

In the following, we obtain some consequences of Theorem 3.13, which permits to extend analogue available results for unbounded (also for continuous) operators on Banach spaces; see [13, 21].

Corollary 3.15. Let $X$ be a complete lcHs and $A \in \mathcal{L}(X)$. If there exists $\Gamma \subseteq \Gamma_{X}$ determining the lc-topology of $X$ such that $A$ is a $\Gamma$-dissipative operator satisfying

$$
\exists c>0 \forall p \in \Gamma \exists q \in \Gamma: p\left(A^{k} x\right) \leq c^{k} q(x) \forall k \in \mathbb{N}, x \in X,
$$

then $A$ generates an equicontinuous $C_{0}$-semigroup on $X$. In particular, $\mathbb{C}^{+} \subseteq \rho(A)$.

Proof. It easily follows via (3.11) that the series $\sum_{k=0}^{\infty} \frac{t^{k} A^{k}}{k !}$ converges in $\mathcal{L}_{b}(X)$ for all $t \geq 0$. So, we can define $T(t):=\sum_{k=0}^{\infty} \frac{t^{k} A^{k}}{k !}$ for all $t \geq 0$, thereby obtaining a $C_{0}$-semigroup of linear continuous operators on $X$. Actually, the semigroup $(T(t))_{t \geq 0}$ is exponentially equicontinuous since, fixed $p \in \Gamma$, by (3.11) we have

$$
p(T(t) x) \leq \sum_{k=0}^{\infty} \frac{t^{k}}{k !} p\left(A^{k} x\right) \leq \sum_{k=0}^{\infty} \frac{c^{k} t^{k}}{k !} q(x)=e^{c t} q(x), \quad x \in X, t \geq 0, p \in \Gamma .
$$

In particular, the operator $(A, X)$ is the infinitesimal generator of $(T(t))_{t \geq 0}$ and so $\{\lambda \in \mathbb{C}: \operatorname{Re} \lambda>$ $c\} \subseteq \rho(A)$; hence, $\operatorname{rg}(\lambda I-A)=X$ for all $\lambda>c$. As $(A, X)$ is dissipative and densely defined, it follows by Theorem 3.13 that $(A, X)$ generates an equicontinuous $C_{0}$-semigroup on $X$ (i.e., the semigroup $(T(t))_{t \geq 0}$ is equicontinuous). Therefore, $\mathbb{C}_{+} \subseteq \rho(A)$. 
Corollary 3.16. Let $(A, D(A))$ be a densely defined operator on a complete lcHs $X$. If both $(A, D(A))$ and $\left(A^{\prime}, D\left(A^{\prime}\right)\right)$ are dissipative operators on $X$ and on $X_{\beta}^{\prime}$ (resp.), then the closure $(\bar{A}, D(\bar{A}))$ of $(A, D(A))$ generates an equicontinuous $C_{0}$-semigroup on $X$.

Proof. By Theorem 3.13 it suffices to show that $\operatorname{rg}(I-A)$ is dense in $X$. So, assume that $\overline{\operatorname{rg}(I-A)} \neq$ $X$. By Hahn-Banach theorem there exists $x^{\prime} \in X^{\prime}$ such that $x^{\prime} \neq 0$ and $\left\langle(I-A) x, x^{\prime}\right\rangle=0$ for all $x \in D(A)$, i.e., $\left\langle x, x^{\prime}\right\rangle=\left\langle A x, x^{\prime}\right\rangle$ for all $x \in D(A)$. Therefore, $x^{\prime} \in D\left(A^{\prime}\right)$ and $A^{\prime} x^{\prime}=x^{\prime}$, i.e., $\left(I-A^{\prime}\right) x^{\prime}=0$. This is a contradiction with the facts that $x^{\prime} \neq 0$ and that $\left(A^{\prime}, D\left(A^{\prime}\right)\right)$ is dissipative; see Proposition 3.10(i).

Theorem 3.17. Let $(A, D(A))$ be a dissipative operator on a sequentially complete lcHs $X$ with $\operatorname{rg}(I-A)=X$. If $X$ is reflexive, then $\overline{D(A)}=X$. If, in addition, $X$ is complete, then $(A, D(A))$ generates an equicontinuous $C_{0}$-semigroup on $X$.

Proof. Let $x^{\prime} \in X^{\prime}$ satisfy $\left\langle x, x^{\prime}\right\rangle=0$ for all $x \in D(A)$. We claim that $x^{\prime}=0$. To this end, as $(I-A)(X)=\operatorname{rg}(I-A)=X$, it suffices to show that $\left\langle x-A x, x^{\prime}\right\rangle=0$ for all $x \in D(A)$. Since $\left\langle x, x^{\prime}\right\rangle=0$ for all $x \in D(A)$, this reduces to show that $\left\langle A x, x^{\prime}\right\rangle=0$ for all $x \in D(A)$.

So, fix $x \in D(A)$. Since $(A, D(A))$ is dissipative and $\operatorname{rg}(I-A)=X$, by Proposition 3.10(iii) we have $\operatorname{rg}(\lambda I-A)=X$ for all $\lambda>0$. Thus, for every $n \in \mathbb{N}$ there is $x_{n} \in D(A)$ such that $n x=(n I-A) x_{n}$. Consequently we have, for every $n \in \mathbb{N}$, that $x=x_{n}-\frac{1}{n} A x_{n}$, i.e., that $A x_{n}=n\left(x_{n}-x\right)$ and hence, $A x_{n} \in D(A)$. Then, for every $n \in \mathbb{N}$ we can apply $A$ to $A x_{n}$ and so we obtain that

$$
A x=A x_{n}-\frac{1}{n} A^{2} x_{n}=\left(I-\frac{1}{n} A\right) A x_{n} .
$$

Which implies that $A x_{n}=\left(I-\frac{1}{n} A\right)^{-1} A x$. Since $(A, D(A))$ is dissipative, by (3.2) we have

$$
p\left(A x_{n}\right)=p\left(\left(I-\frac{1}{n} A\right)^{-1} A x\right) \leq p(A x), \quad n \in \mathbb{N}, p \in \Gamma,
$$

where $\Gamma \subseteq \Gamma_{X}$ generates the lc-topology of $X$. Then, we have

$$
p\left(x_{n}-x\right)=\frac{1}{n} p\left(A x_{n}\right) \leq \frac{1}{n} p(A x), \quad n \in \mathbb{N}, p \in \Gamma,
$$

and so, $x_{n} \rightarrow x$ in $X$.

From (3.12) we obtain that the set $B:=\left\{A x_{n}: n \in \mathbb{N}\right\}$ is bounded in $X$ and hence, by the reflexivity of $X, B$ is a relatively $\sigma\left(X, X^{\prime}\right)$-compact subset of $X$. Consequently, $B$ has a $\sigma\left(X, X^{\prime}\right)$-cluster point in $X$, say $y \in X$. So, the set $\left\{\left(x_{n}, A x_{n}\right): n \in \mathbb{N}\right\} \subseteq \mathcal{G}(A)$ has $(x, y)$ as a $\sigma\left(X \times X, X^{\prime} \times X^{\prime}\right)$-cluster point. Now, we see that $(x, y) \in \mathcal{G}(A)$ and so $A x=y$. Indeed, as $(A, D(A))$ is dissipative and $\operatorname{rg}(I-A)=X$ (hence, $\operatorname{rg}(I-A)$ is closed in $X)$, by Proposition 3.10(ii) we have that $(A, D(A))$ is closed, and so that $\mathcal{G}(A)$ is closed in $X \times X$. The fact that $\mathcal{G}(A)$ is a closed subspace of $X \times X$ yields that $\mathcal{G}(A)$ is also $\sigma\left(X \times X, X^{\prime} \times X^{\prime}\right)$-closed in $X \times X$. Therefore, every $\sigma\left(X \times X, X^{\prime} \times X^{\prime}\right)$-cluster point of $\mathcal{G}(A)$ necessarily belongs to $\mathcal{G}(A)$.

Since $\left\langle w, x^{\prime}\right\rangle=0$ for all $w \in D(A)$ and $x \in D(A),\left(x_{n}\right)_{n \in \mathbb{N}} \subseteq D(A)$, we have $\left\langle x_{n}, x^{\prime}\right\rangle=\left\langle x-x_{n}, x^{\prime}\right\rangle=$ 0 for all $n \in \mathbb{N}$. So, as $A x_{n}=n\left(x-x_{n}\right)$ for all $n \in \mathbb{N}$, it follows that

$$
\left\langle A x_{n}, x^{\prime}\right\rangle=\left\langle n\left(x-x_{n}\right), x_{n}\right\rangle=n\left\langle x-x_{n}, x^{\prime}\right\rangle=0
$$

for all $n \in \mathbb{N}$. Since $A x$ is a $\sigma\left(X, X^{\prime}\right)$-cluster point of $\left(A x_{n}\right)_{n \in \mathbb{N}}$, we get that $\left\langle A x, x^{\prime}\right\rangle=0$. This completes the proof because we have shown that $\left\langle x-A x, x^{\prime}\right\rangle=0$, for all $x \in D(A)$, i.e., $\left\langle z, x^{\prime}\right\rangle=0$ for all $z \in \operatorname{rg}(I-A)=X$. This implies that $x=0$. So, we can conclude that $\overline{D(A)}=X$.

Now, suppose that $X$ is also complete. Then the fact that $(A, D(A))$ is a dissipative, densely defined operator on $X$ with $\operatorname{rg}(I-A)=X$ implies by Theorem 3.13 that $(A, D(A))$ generates an equicontinuous $C_{0}$-semigroup on $X$. 


\section{Dissipative operators and additive perturbations}

In this section we first adapt the notion of subdifferential to the setting of locally convex spaces along the lines of the classical theory of unbounded operators acting in Banach spaces; see, f.i., [9], [13]. Later, we present some additive perturbation results of dissipative operators on lcHs.

Let $X$ be a lcHs. For each $p \in \Gamma_{X}$, set $U_{p}:=\{x \in X: p(x) \leq 1\}$ and define the dual seminorm $p^{\prime}$ of $p$ on $X^{\prime}$ by

$$
p^{\prime}\left(x^{\prime}\right):=\sup \left\{\left|\left\langle x, x^{\prime}\right\rangle\right|: p(x) \leq 1\right\}=\sup \left\{\left|\left\langle x, x^{\prime}\right\rangle\right|: p(x)=1\right\}, \quad x^{\prime} \in X^{\prime},
$$

i.e., $p^{\prime}$ is the gauge of the polar $U_{p}^{\circ}$ in $X^{\prime}$. Set $X_{p}:=(X / \operatorname{Ker} p, \hat{p})$ (where $\hat{p}$ denotes the norm induced on $X / \operatorname{Ker} p$ by $p)$ and $X_{p}^{\prime}:=\left\{x^{\prime} \in X^{\prime}: p^{\prime}\left(x^{\prime}\right)<\infty\right\}$. Then $X_{p}$ is a normed space and $\left(X_{p}^{\prime}, p^{\prime}\right)$ is a Banach space. In particular, if $Q_{p}: X \rightarrow X_{p}$ denotes the canonical quotient map from $X$ onto $X_{p}$, then its dual map $Q_{p}^{\prime}$ is an isometry from the strong dual of the normed space $X_{p}$ onto $\left(X_{p}^{\prime}, p^{\prime}\right)$. Therefore, every $x^{\prime} \in(X / \operatorname{Ker} p, \hat{p})^{\prime}$ defines a continuous linear functional $\bar{x}^{\prime}=x^{\prime} \circ Q_{p} \in X^{\prime}$ with $p^{\prime}\left(\bar{x}^{\prime}\right)<\infty$. In particular, $\left|\left\langle x, \bar{x}^{\prime}\right\rangle\right|=\left|x^{\prime}\left(Q_{p} x\right)\right| \leq p^{\prime}\left(\bar{x}^{\prime}\right) p(x)$, for all $x \in X$.

Let $X$ be a real or complex lcHs. For each $p \in \Gamma_{X}$ and $x \in X$, we set

$$
d p(x):=\left\{x^{\prime} \in X^{\prime}: \operatorname{Re}\left\langle y, x^{\prime}\right\rangle \leq p(y) \forall y \in X,\left\langle x, x^{\prime}\right\rangle=p(x)\right\} .
$$

The set $d p(x)$ is called subdifferential of $p$ in $x$.

Observe that $d p(x) \neq \emptyset$ for all $x \in X$ and $p \in \Gamma_{X}$. Indeed, if $p(x) \neq 0$, then the result follows from the Hahn-Banach theorem. In case $p(x)=0$, consider the normed space $X_{p}$ and its topological dual $\left(X_{p}^{\prime}, p^{\prime}\right)$. Then, for every $x^{\prime} \in X_{p}^{\prime}$, the composition map $\bar{x}^{\prime}:=x^{\prime} \circ Q_{p}$ belongs to $X^{\prime}$ and satisfies $\bar{x}^{\prime}(x)=0=p(x)$. Moreover, we have

$$
\operatorname{Re}\left\langle y, \bar{x}^{\prime}\right\rangle \leq\left|\left\langle y, \bar{x}^{\prime}\right\rangle\right|=\left|\left\langle y, x^{\prime} \circ Q_{p}\right\rangle\right|=\left|x^{\prime}\left(Q_{p} y\right)\right| \leq c p\left(Q_{p} y\right) \mid \leq c p(y),
$$

for all $y \in X$ and some constant $c>0$. Accordingly, $c^{-1} \bar{x}^{\prime} \in d p(x)$ and so $d p(x) \neq \emptyset$.

Example 4.1. 1. Let $\Omega \subseteq \mathbb{R}^{N}$ be an open set and let $X:=C(\Omega)=\{f: \Omega \rightarrow \mathbb{C}: f$ continuous on $\Omega\}$. Suppose that $\left(K_{h}\right)_{h}$ is sequence of compact subsets of $\Omega$ such that $K_{h}=\stackrel{\circ}{K^{\circ}} \subseteq \stackrel{\circ}{K^{\prime}} h+1 \subseteq \Omega$ and $\cup_{h} K_{h}=\Omega$ and define $p_{h}(f):=\max _{x \in K_{h}}|f(x)|, h \in \mathbb{N}$ and $f \in X$.

Then $X$ is a Fréchet space with respect to the lc-topology generated by $\left(p_{h}\right)_{h}$. In particular, for every $h \in \mathbb{N}$ and $f \in X$ with $f \neq 0$ on $K_{h}$, we have

$$
\left\{\operatorname{sign} f\left(x_{0}\right) \cdot \delta_{x_{0}}: x_{0} \in K_{h} \text { and } p_{h}(f)=\left|f\left(x_{0}\right)\right|\right\} \subseteq d p_{h}(f)
$$

(recall that, for $z \in \mathbb{C}, \operatorname{sign} z=\frac{\bar{z}}{|z|}$ if $z \neq 0$ and $\operatorname{sign} z=0$ if $z=0$ ). In fact, for fixed $h \in \mathbb{N}$ af $\in X$ with $f \neq 0$ on $K_{h}$ and $x_{0} \in K_{h}$ such that $p_{h}(f)=\left|f\left(x_{0}\right)\right|$, the linear functional $F(g):=\operatorname{sign} f\left(x_{0}\right) g\left(x_{0}\right)$, for $g \in X$, belongs to $X^{\prime}$ because $\operatorname{Re} F(g) \leq|F(g)|=\left|g\left(x_{0}\right)\right| \leq p_{h}(g)$ for all $g \in X$. Moreover, $F(f)=\operatorname{sign} f\left(x_{0}\right) f\left(x_{0}\right)=\left|f\left(x_{0}\right)\right|=p_{h}(f)$.

In case $f=0$ on $K_{h}$ and so $p_{h}(f)=0$, for every $F \in X_{p_{h}}^{\prime}$ the linear functional $\bar{F}=F \circ Q_{p_{h}}$ belongs to $X^{\prime}$ and satisfies $\bar{F}(f)=0=p_{h}(f)$ and $\operatorname{Re} \bar{F}(g) \leq c p_{h}(g)$ for all $g \in X$ and some constant $c>0$. So, $c^{-1} \bar{F} \in d p_{h}(f)$.

2. Let $1 \leq p<\infty$ and $\Omega \subseteq \mathbb{R}^{N}$ be an open set. The space $X^{p}:=L_{l o c}^{p}(\Omega)$ of all locally $p$-integrable functions on $\Omega$ is a Fréchet space with respect to the lc-topology given by the sequence of seminorms

$$
\|f\|_{p, h}:=\left(\int_{K_{h}}|f(x)|^{p} d x\right)^{1 / p}, \quad h \in \mathbb{N}, f \in X^{p},
$$

where $\left(K_{h}\right)_{h}$ is sequence of compact subsets of $\Omega$ such that $K_{h}=\overline{\stackrel{\circ}{K}} \stackrel{\circ}{\leftarrow}_{h}{ }_{h+1} \subseteq \Omega$ and $\cup_{h} K_{h}=\Omega$.

If $1<p<\infty$ then, for every $h \in \mathbb{N}$ and $f \in X^{p}$ with $f \neq 0$ on $K_{h}$, the function $\varphi: \Omega \rightarrow \mathbb{C}$ defined by

$$
\varphi(x):= \begin{cases}\overline{f(x)}|f(x)|^{p-2}\|f\|_{p, h}^{1-p} & \text { if } x \in K_{h}, f(x) \neq 0 \\ 0 & \text { otherwise }\end{cases}
$$


determines an element of $d\|\|_{p, h}(f)$. To see this, fix $h \in \mathbb{N}$ and $f \in X^{p}$ with $f \neq 0$ on $K_{h}$. We have that the linear functional $F(g):=\int_{\Omega} \varphi(x) g(x) d x$, for $g \in X^{p}$, satisfies

$$
\begin{aligned}
\operatorname{Re} F(g) & \leq|F(g)| \leq \int_{K_{h}}|\varphi(x) \| g(x)| d x \leq\left(\int_{K_{h}}|\varphi(x)|^{q} d x\right)^{1 / q} \cdot\left(\int_{K_{h}}|g(x)|^{p} d x\right)^{1 / p} \\
& =\|f\|_{p, h}^{1-p}\left(\int_{K_{h}}|f(x)|^{(p-1) q} d x\right)^{1 / q}\|g\|_{p, h}=\|f\|_{p, h}^{1-p}\|f\|_{p, h}^{p-1}\|g\|_{p, h}=\|g\|_{p, h}, \quad g \in X^{p},
\end{aligned}
$$

where $q$ satisfies $\frac{1}{p}+\frac{1}{q}=1$ and hence $(p-1) q=p$ and $\frac{p}{q}=p-1$. Moreover, we have

$$
F(f)=\int_{\Omega} \varphi(x) f(x) d x=\|f\|_{p, h}^{1-p} \int_{K_{h}}|f(x)|^{p} d x=\|f\|_{p, h}^{1-p}\|f\|_{p, h}^{p}=\|f\|_{p, h} .
$$

Let $p=1$. Then, for every $h \in \mathbb{N}$ and $f \in X^{1}$ with $f \neq 0$ on $K_{h}$, the function $\varphi: \Omega \rightarrow \mathbb{C}$ defined by

$$
\varphi(x):= \begin{cases}\operatorname{sign} f(x) & \text { if } x \in K_{h}, f(x) \neq 0 \\ 0 & \text { otherwise, }\end{cases}
$$

determines an element of $d\|\|_{1, h}(f)$. Indeed, for fixed $h \in \mathbb{N}$ and $f \in X^{1}$ with $f \neq 0$ on $K_{h}$, the linear functional $F(g):=\int_{\Omega} \varphi(x) g(x) d x$, for $g \in X^{1}$, satisfies

$$
\operatorname{Re} F(g) \leq|F(g)| \leq \int_{K_{h}}|g(x)| d x=\|g\|_{1, h}, \quad g \in X^{1},
$$

and

$$
F(f)=\int_{K_{h}}|f(x)| d x=\|f\|_{1, h} .
$$

In case $f=0$ on $K_{h}$, for every $1 \leq p<\infty$ the set $d\|\|_{p, h}(f)$ contains all the elements of type $\bar{F}:=F \circ Q_{\|\|_{p, h}}$ with $F \in\left(X_{\|\|_{p, h}}^{p}\right)^{\prime}$ of norm $\leq 1$.

Proposition 4.2. Let $(A, D(A))$ be an operator on a lcHs $X$. Then $(A, D(A))$ is dissipative if and only if there exists $\Gamma \subseteq \Gamma_{X}$ determining the lc-topology of $X$ such that for every $p \in \Gamma$ and $x \in D(A)$, there exists $x^{\prime} \in d p(x)$ satisfying $\operatorname{Re}\left\langle A x, x^{\prime}\right\rangle \leq 0$.

Proof. Suppose that $(A, D(A))$ is dissipative. Then there exists $\Gamma \subseteq \Gamma_{X}$ determining the lc-topology of $X$ such that the inequalities in (3.1) are satisfied.

Fixed $p \in \Gamma$ and $x \in D(A)$, for each $t>0$ we choose $x_{t}^{\prime} \in d p(x-t A x)$. Then, for every $t>0$ and $y \in X$ we have $\operatorname{Re}\left\langle y, x_{t}^{\prime}\right\rangle \leq p(y)$. Since $\left|\left\langle y, x_{t}^{\prime}\right\rangle\right|=\alpha\left\langle y, x_{t}^{\prime}\right\rangle$ for some $\alpha \in \mathbb{C}$ with $|\alpha|=1$, we get that $\left|\left\langle y, x_{t}^{\prime}\right\rangle\right|=\left\langle\alpha y, x_{t}^{\prime}\right\rangle=\operatorname{Re}\left\langle\alpha y, x_{t}^{\prime}\right\rangle \leq p(\alpha y)=p(y)$ for all $t>0, y \in X$. Hence, the net $\left\{x_{t}^{\prime}: t>0\right\} \subset X_{p}^{\prime}$ is bounded in $X_{p}^{\prime}$. Consequently, it possesses a $\sigma\left(X_{p}^{\prime}, X_{p}\right)$-limit point $x^{\prime} \in X_{p}^{\prime}$ as $t \rightarrow 0$ and so a $\sigma\left(X^{\prime}, X\right)$-limit point in $X^{\prime}$, say $x^{\prime}$, as $t \rightarrow 0$.

We claim that $x^{\prime} \in d p(x)$ and $\operatorname{Re}\left\langle A x, x^{\prime}\right\rangle \leq 0$. Indeed, since $\operatorname{Re}\left\langle y, x_{t}^{\prime}\right\rangle \leq p(y)$, for all $t>0$ and $y \in X$, it follows by passing to the limit for $t \rightarrow 0$ that $\operatorname{Re}\left\langle y, x^{\prime}\right\rangle \leq p(y)$ for all $y \in X$. On the other hand, from $\left\langle y, x_{t}^{\prime}\right\rangle-t\left\langle A x, x_{t}^{\prime}\right\rangle=\left\langle y-t A x, x_{t}^{\prime}\right\rangle=p(x-t A x)$, for all $t>0$, we get by passing to the limit for $t \rightarrow 0$ that $\left\langle x, x^{\prime}\right\rangle=p(x)$. Hence, $x^{\prime} \in d p(x)$.

Since $(A, D(A))$ is dissipative and $x^{\prime} \in d p(x)$, we obtain, for every $t>0$, that

$$
\begin{aligned}
p(x) & \leq p(x-t A x)=\left\langle x-t A x, x_{t}^{\prime}\right\rangle=\operatorname{Re}\left\langle x-t A x, x_{t}^{\prime}\right\rangle \\
& =\operatorname{Re}\left\langle x, x_{t}^{\prime}\right\rangle-t \operatorname{Re}\left\langle A x, x_{t}^{\prime}\right\rangle \leq p(x)-t \operatorname{Re}\left\langle A x, x_{t}^{\prime}\right\rangle .
\end{aligned}
$$

Accordingly, $\operatorname{Re}\left\langle A x, x_{t}^{\prime}\right\rangle \leq 0$ for all $t>0$, which implies by passing to the limit for $t \rightarrow 0$ that $\operatorname{Re}\left\langle A x, x^{\prime}\right\rangle \leq 0$. So, the claim is proved.

To show the converse, fix $p \in \Gamma, x \in D(A)$ and $t>0$. Then by assumption, there exists $x^{\prime} \in d p(x)$ satisfying $\operatorname{Re}\left\langle A x, x^{\prime}\right\rangle \leq 0$. It follows that

$$
\begin{aligned}
p(x) & =\left\langle x, x^{\prime}\right\rangle=\operatorname{Re}\left\langle x, x^{\prime}\right\rangle=\operatorname{Re}\left\langle x-t A x+t A x, x^{\prime}\right\rangle=\operatorname{Re}\left\langle x-t A x, x^{\prime}\right\rangle+t \operatorname{Re}\left\langle A x, x^{\prime}\right\rangle \leq \\
& \leq \operatorname{Re}\left\langle x-t A x, x^{\prime}\right\rangle \leq p(x-t A x)
\end{aligned}
$$


and hence, that

$$
p\left(\left(\frac{1}{t} I-A\right) x\right) \geq \frac{1}{t} p(x), \text { i.e., } p((\lambda I-A) x) \geq \lambda p(x) \text { for } \lambda=\frac{1}{t} .
$$

Accordingly, $(A, D(A))$ is dissipative.

Example 4.3. Let $\left.\left.\mathbb{R}_{-}:=\right]-\infty, 0\right]$ and let $X:=C((-\infty, 0])$ be the Fréchet space of all complex-valued continuous functions on $\mathbb{R}_{-}$endowed with the lc-topology generated by the sequence of seminorms $p_{h}(f):=\max _{x \in[-h, 0]}|f(x)|$, for $h \in \mathbb{N}$ and $f \in X$. Consider the operator

$$
\text { Af }:=f^{\prime}, \quad f \in D(A):=\left\{f \in C^{1}\left(\mathbb{R}_{-}\right): f^{\prime}(0)=L f\right\},
$$

where $L \in X^{\prime}$ and so there exists $h_{0} \in \mathbb{N}$ such that $p_{h_{0}}^{\prime}(L)=\sup _{p_{h_{0}}(f) \leq 1}|L(f)|<\infty$. The operator defined in (4.1) is the so-called delay differential operator.

If we define $\varphi(f):=f^{\prime}(0)-L f$, for $f \in C^{1}\left(\mathbb{R}_{-}\right)$, and endow the space $Y:=C^{1}\left(\mathbb{R}_{-}\right)$with the lc-topology generated by the sequence of seminorms $q_{h}(f):=\max _{x \in[-h, 0]}|f(x)|+\max _{x \in[-h, 0]}\left|f^{\prime}(x)\right|$, for $h \in \mathbb{N}$ and $f \in Y$, then $\varphi \in Y^{\prime}$ and $D(A)=\operatorname{Ker} \varphi$. So, $D(A)$ is a closed subspace of $Y$. On the other hand, $\varphi \notin X^{\prime}$ and hence, $D(A)$ is a dense subspace of $X$.

Now, we show that the operator $\left(A-p_{h_{0}}^{\prime}(L) I, D(A)\right)$ is dissipative in $X$. To see this, fix $h \in \mathbb{N}$ with $h \geq h_{0}$ and $f \in D(A)$ with $f \neq 0$ on $K_{h}:=[-h, 0]$. As in Example 4.1 one shows that the linear functional $\operatorname{sign} f\left(x_{0}\right) \delta_{x_{0}}$ belongs to $d p_{h}(f)$ whenever $x_{0} \in K_{h}$ satisfies $p_{h}(f)=\left|f\left(x_{0}\right)\right|$. In such a case, we have

$$
\operatorname{Re}\left\langle A f-p_{h_{0}}^{\prime}(L) f, \operatorname{sign} f\left(x_{0}\right) \delta_{x_{0}}\right\rangle=\operatorname{Re} \operatorname{sign} f\left(x_{0}\right) f^{\prime}\left(x_{0}\right)-\left|f\left(x_{0}\right)\right| p_{h_{0}}^{\prime}(L) \leq 0
$$

if and only if

$$
\operatorname{Re} \operatorname{sign} f\left(x_{0}\right) f^{\prime}\left(x_{0}\right) \leq p_{h}(f) p_{h_{0}}^{\prime}(L),
$$

being $p_{h}(f)=\left|f\left(x_{0}\right)\right|$. If $-h<x_{0}<0$, then Resign $f\left(x_{0}\right) f^{\prime}\left(x_{0}\right)=0$ and so the inequality in (4.3) holds. In the case $x_{0}=-h$, we have

$$
\operatorname{Resign} f\left(x_{0}\right) f^{\prime}\left(x_{0}\right)=\frac{1}{\left|f\left(x_{0}\right)\right|} \operatorname{Re} \overline{f\left(x_{0}\right)} f^{\prime}\left(x_{0}\right)=\frac{1}{2\left|f\left(x_{0}\right)\right|} \operatorname{Re}(\bar{f} \cdot f)^{\prime}\left(x_{0}\right) \leq 0
$$

and so the inequality in (4.3) holds. Finally, if $x_{0}=0$, then

$$
\operatorname{Re} \operatorname{sign} f\left(x_{0}\right) f^{\prime}\left(x_{0}\right)=\operatorname{Re} \operatorname{sign} f\left(x_{0}\right) L f \leq\left|\operatorname{sign} f\left(x_{0}\right)\right||L f|=|L f| \leq p_{h_{0}}(f) p_{h_{0}}^{\prime}(L) \leq p_{h}(f) p_{h_{0}}^{\prime}(L) .
$$

Thus, the inequality in (4.3) holds.

In case $f=0$ on $K_{h}$ and so $f^{\prime}=0$ on $K_{h}$ too, every element of type $\bar{F}:=F \circ Q_{p_{h}}$ with $F \in X_{p_{h}}^{\prime}$ of norm $\leq 1$ belongs to $d p_{h}(f)$ and satisfies

$$
\operatorname{Re}\left\langle A f-p_{h_{0}}^{\prime}(L) f, \bar{F}\right\rangle=\operatorname{Re}\left\langle Q_{p_{h}} f^{\prime}-p_{h_{0}}^{\prime}(L) Q_{p_{h}} f, F\right\rangle=\operatorname{Re}\langle 0, F\rangle=0,
$$

being $Q_{p_{h}}$ the canonical quotient map from $X$ onto $X_{p_{h}}=X / \operatorname{Ker} p_{h}$.

Since $\left(p_{h}\right)_{h \geq h_{0}}$ generates the lc-topology of $X$ also, we can conclude via Proposition 4.2 that the operator $\left(\left(A-p_{h_{0}}^{\prime}(L) I\right), D(A)\right)$ is dissipative in $X$.

The operator $\left(\lambda I-\left(A-p_{h_{0}}^{\prime}(L) I\right), D(A)\right)$ is also surjective for every $\lambda>0$. Indeed, fixed $\lambda>0$ and $g \in X$, if we set $\mu:=\lambda+p_{h_{0}}^{\prime}(L)$, then the function

$$
f(x):=c e^{\mu}-\int_{0}^{x} e^{\mu(x-y)} g(y) d y=: c \epsilon_{\mu}(x)+K(x), \quad x \leq 0,
$$

with $c:=\frac{g(0)-L K}{\mu-L \epsilon_{\mu}}$, satisfies the equations $\lambda f-\left(A-p_{h_{0}}^{\prime}(L) I\right) f=g$ and $f^{\prime}(0)=L f$; see, f.i., [13, Ch. II, 3.29].

Therefore, by Theorem 3.13 the operator $\left(\left(A-p_{h_{0}}^{\prime}(L) I\right), D(A)\right)$ generates an equicontinuous $C_{0^{-}}$ semigroup $(T(t))_{t \geq 0}$ on $X$, i.e., $p_{h}(T(t) f) \leq p_{h}(f)$ for all $f \in X$ and $h \in \mathbb{N}$. In particular, the rescaled $C_{0}$-semigroup $S(t):=e^{p_{h_{0}}^{\prime}(L) t} T(t), t \geq 0$, satisfies

$$
p_{h}(S(t) f) \leq e^{p_{h_{0}}^{\prime}(L) t} p_{h}(f), \quad f \in X, h \in \mathbb{N} .
$$


Moreover, its infinitesimal generator coincides with $(A, D(A))$.

The next result is also available.

Proposition 4.4. Let $X$ be a complete lcHs. Let $(A, D(A))$ be the infinitesimal generator of an exponentially equicontinuous $C_{0}$-semigroup $(T(t))_{t>0}$ on $X$. Then the following assertions are equivalent.

(i) $(T(t))_{t \geq 0}$ is equicontinuous.

(ii) There exists $\Gamma \subseteq \Gamma_{X}$ generating the lc-topology of $X$ such that for every $p \in \Gamma, x \in D(A)$ and $x^{\prime} \in d p(x)$, we have $\operatorname{Re}\left\langle A x, x^{\prime}\right\rangle \leq 0$.

Proof. (i) $\Rightarrow\left(\right.$ ii). Since $(T(t))_{t \geq 0}$ is equicontinuous, by [5, Remark 2.2(i)] there exists $\Gamma \subseteq \Gamma_{X}$ generating the lc-topology of $X$ such that

$$
p(T(t) x) \leq p(x), \quad p \in \Gamma, x \in X, t \geq 0 .
$$

So, fixed any $p \in \Gamma, x \in D(A)$ and $x^{\prime} \in d p(x)$, it follows, for every $t>0$, that

$$
\begin{aligned}
\operatorname{Re}\left\langle\frac{T(t) x-x}{t}, x^{\prime}\right\rangle & =\frac{1}{t}\left(\operatorname{Re}\left\langle T(t) x, x^{\prime}\right\rangle-\operatorname{Re}\left\langle x, x^{\prime}\right\rangle\right) \\
& \leq \frac{1}{t}(p(T(t) x)-p(x)) \leq \frac{1}{t}(p(x)-p(x))=0 .
\end{aligned}
$$

Then

$$
\operatorname{Re}\left\langle A x, x^{\prime}\right\rangle=\lim _{t \rightarrow 0+} \operatorname{Re}\left\langle\frac{T(t) x-x}{t}, x^{\prime}\right\rangle \leq 0 .
$$

So, since $p \in \Gamma$ and $x \in D(A)$ are arbitrary, (ii) follows.

(ii) $\Rightarrow(\mathrm{i})$. Since $(A, D(A))$ generates an exponentially equicontinuous $C_{0}$-semigroup $(T(t))_{t>0}$ on $X$ and $X$ is complete, the operator $(A, D(A))$ is closed and $(w, \infty) \subseteq \rho(A)$ for some $w \in \mathbb{R}$. Hence, there exists $\lambda>0$ such that $\operatorname{rg}(\lambda I-A)=X$. On the other hand, the assumption implies that the operator $(A, D(A))$ is dissipative; see Proposition 4.2. So, we can apply Theorem 3.13 to conclude that the semigroup $\left((T(t))_{t \geq 0}\right.$ is equicontinuous.

In what follows, some further notations are required. Let $X$ be a lcHs and let $\Gamma \subseteq \Gamma_{X}$ determine the lc-topology of $X$. An operator $T \in \mathcal{L}(X)$ is said to be $\Gamma$-contractive if, for every $p \in \Gamma$ and $x \in X$, we have $p(T x) \leq p(x)$. Accordingly, a semigroup $(T(t))_{t>0} \subseteq \mathcal{L}(X)$ is called $\Gamma$-contractively equicontinuous if, for every $p \in \Gamma, x \in X$ and $t>0$, we have $p(T(t) x) \leq p(x)$. A power bounded operator $T \in \mathcal{L}(X)$ (i.e., such that $\left\{T^{n}\right\}_{n \in \mathbb{N}}$ is equicontinuous) is $\Gamma$-contractive for some $\Gamma \subseteq \Gamma_{X}$ which determines the lc-topology of $X$; see, f.i., [8, Lemma 18]. Analogously, an equicontinuous semigroup $(T(t))_{t>0}$ on $X$ is always $\Gamma$-contractively equicontinuous for some $\Gamma \subseteq \Gamma_{X}$ with $\Gamma$ generating the lc-topology of $X$; see, f.i., [5, Remark 2.2(i)].

Proposition 4.5. Let $X$ be a complete lcHs. Let $(A, D(A))$ be the infinitesimal generator of a $\Gamma$ contractively equicontinuous $C_{0}$-semigroup on $X$ for some $\Gamma \subseteq \Gamma_{X}$ determining the lc-topology of $X$. Let $(B, D(B))$ be a $\Gamma$-dissipative operator on $X$ such that $D(A) \subseteq D(B)$. If there exists $\lambda>0$ such that

$$
\operatorname{rg}(\lambda I-(A+B))=X,
$$

then $(A+B, D(A))$ generates a $\Gamma$-contractively equicontinuous $C_{0}$-semigroup on $X$.

Proof. Since $(A, D(A))$ generates a $\Gamma$-contractively equicontinuous $C_{0}$-semigroup on $X$, the operator $(A, D(A))$ is densely defined and closed on $X$. Moreover, we have that $\operatorname{Re}\left\langle A x, x^{\prime}\right\rangle \leq 0$ for all $p \in \Gamma$, $x \in D(A)$ and $x^{\prime} \in d p(x)$; see Proposition $4.4(\mathrm{i}) \Rightarrow(\mathrm{ii})$. On the other hand, as $(B, D(B))$ is $\Gamma$ dissipative, by Proposition 4.2 we have, for every $p \in \Gamma$ and $x \in D(B)$, that there exists $\bar{x}^{\prime} \in d p(x)$ such that $\operatorname{Re}\left\langle B x, x^{\prime}\right\rangle \leq 0$. Then it follows, for every $p \in \Gamma$ and $x \in D(A)(D(A) \subseteq D(B))$, that there exists $\bar{x}^{\prime} \in d p(x)$ such that $\operatorname{Re}\left\langle(A+B) x, x^{\prime}\right\rangle \leq 0$. Accordingly, the operator $(A+B, D(A))$ is $\Gamma$-dissipative. As $\operatorname{rg}(\lambda I-(A+B))=X$, it follows via Proposition 3.10(ii) that the operator $(A+B, D(A))$ is also closed. 
Since $(A+B, D(A))$ is a $\Gamma$-dissipative, closed densely defined operator on $X$ with $\operatorname{rg}(\lambda I-(A+B))=$ $X$, for some $\lambda>0$, and $X$ is complete, by Theorem 3.13 the result follows, i.e., $(A+B, D(A))$ generates a $\Gamma$-contractively equicontinuous $C_{0}$-semigroup on $X$.

Example 4.6. Let $X:=\{f:] 0,+\infty\left[\rightarrow \mathbb{C}: f \in C(] 0,+\infty[), \exists \lim _{x \rightarrow 0^{+}} x f(x) \in \mathbb{C}\right\}$. Then $X$ is a Fréchet space with respect the lc-topology generated by the seminorms $p_{h}(f)=\sup _{x \in[0, h]} x|f(x)|$, for $h \in \mathbb{N}$ and $f \in X$.

We consider the operator $A f(x):=-x f^{\prime}(x)-f(x)$, for $x>0$ and $f \in D(A):=\{g:] 0,+\infty[\rightarrow$ $\left.\mathbb{C}: g \in X \cap C^{1}(] 0,+\infty[), \exists \lim _{x \rightarrow 0^{+}} x^{2} g^{\prime}(x) \in \mathbb{C}\right\}$. Then $(A, D(A))$ is an operator on $X$. Indeed, for every $f \in D(A)$, we have $f \in C(] 0,+\infty[)$ and $\lim _{x \rightarrow 0^{+}} x A f(x)=\lim _{x \rightarrow 0^{+}}\left(-x^{2} f^{\prime}(x)-x f(x)\right)$ exists in $\mathbb{C}$. Moreover, we will see that the operator $(A, D(A))$ is dissipative on $X$ with $\operatorname{rg}(\lambda I-A)=X$ for all $\lambda>0$. To this aim, fix $\lambda>0$ and $g \in X$ and consider the equation $\lambda f-A f=g$ on $] 0,+\infty[$, i.e., $(\lambda+1) f(x)+x f^{\prime}(x)=g(x)$ for $x>0$. An easy calculation yields that the solutions are given by

$$
f(x)=x^{-(\lambda+1)} \int_{0}^{x} y^{\lambda} g(y) d y+c x^{-(\lambda+1)}=: F(x)+c x^{-(\lambda+1)}, \quad x>0, c \in \mathbb{C} .
$$

Since $g \in X$ and hence, $g \in C(] 0,+\infty[)$ and $\lim _{x \rightarrow 0^{+}} x g(x)$ exists in $\mathbb{C}$, the definite integral on the right in (4.5) converges. Indeed, from the existence of $\lim _{x \rightarrow 0^{+}} x g(x)$ in $\mathbb{C}$, it follows that the function $x \in] 0, \infty[\mapsto x g(x)$ is bounded in a right neighbourhood of 0 , say in $] 0, \delta]$, and so

$$
\left|\int_{0}^{\delta} y^{\lambda} g(y) d y\right| \leq \int_{0}^{\delta} y^{\lambda-1} y|g(y)| d y \leq M \int_{0}^{\delta} y^{\lambda-1} d y=M \frac{\delta^{\lambda}}{\lambda},
$$

where $M:=\sup _{y \in[0, \delta]} y|g(y)|$. Consequently, we can conclude that $f \in C^{1}(] 0, \infty[)$.

Now, by L'Hôpital rule we obtain

$$
\lim _{x \rightarrow 0^{+}} x F(x)=\lim _{x \rightarrow 0^{+}} \frac{\int_{0}^{x} y^{\lambda} g(y) d y}{x^{\lambda}}=\lim _{x \rightarrow 0^{+}} \frac{x^{\lambda} g(x)}{\lambda x^{\lambda-1}}=\frac{1}{\lambda} \lim _{x \rightarrow 0^{+}} x g(x),
$$

and hence, the function given in (4.5) defines an element of the space $X$ if and only if $c=0$. In such a case $f^{\prime}(x)=-(\lambda+1) x^{-(\lambda+2)} \int_{0}^{x} y^{\lambda} g(y) d y+\frac{g(x)}{x}$, for $x>0$. So

$$
\lim _{x \rightarrow 0^{+}} x^{2} f^{\prime}(x)=\lim _{x \rightarrow 0^{+}}\left[-(\lambda+1) \frac{\int_{0}^{x} y^{\lambda} g(y) d y}{x^{\lambda}}+x g(x)\right]=-\frac{1}{\lambda} \lim _{x \rightarrow 0^{+}} x g(x) .
$$

Therefore, the function $f$ given in (4.5) with $c=0$ belongs to $D(A)$. Moreover, for a fixed $h \in \mathbb{N}$, from (4.5) with $c=0$ it follows, for each $x \in] 0, h]$, that

$$
x|f(x)| \leq x^{-\lambda} \int_{0}^{x} y^{\lambda}|g(y)| d y \leq p_{h}(g) x^{-\lambda} \int_{0}^{x} y^{\lambda-1} d y=\frac{1}{\lambda} p_{h}(g)
$$

and hence, that

$$
p_{h}(f) \leq \frac{1}{\lambda} p_{h}(g)=\frac{1}{\lambda} p_{h}((\lambda I-A) f) .
$$

Since $g \in X, \lambda>0$ and $h \in \mathbb{N}$ are arbitrary, via (4.7) we can conclude that the operator $(A, D(A))$ is dissipative with $\operatorname{rg}(\lambda I-A)=X$, for every $\lambda>0$. In particular, the domain $D(A)$ is dense in $X$. Indeed, set $Z:=\operatorname{span}\left\{x^{n}: n \geq-1\right\}$, we have that $Z \subseteq D(A)$ and $Z$ is dense in $X$. To see this, fix $f \in X, h \in \mathbb{N}$ and $\varepsilon>0$. Since $f \in C(] 0,+\infty[)$ and $\lim _{x \rightarrow 0^{+}} x f(x)$ exists in $\mathbb{C}$, the function $x \in$ ] $0,+\infty\left[\mapsto x f(x)\right.$ can be continuously extended at 0 and so there exists a polynomial $g(x)=\sum_{r=0}^{l} c_{r} x^{r}$ with complex coefficients such that $\max _{x \in[0, h]}|x f(x)-g(x)|<\varepsilon$. But, $\max _{x \in[0, h]}|x f(x)-g(x)|=$ $p_{h}\left(f-x^{-1} g\right)$ with $x^{-1} g \in Z$. Therefore, $Z$ is a dense subspace of $X$. Consequently, by Theorem 3.13 we can conclude that the operator $(A, D(A))$ generates an equicontinuous $C_{0}$-semigroup in $X$.

Next, we consider the operator $B f(x):=-x f(x)$, for $x>0$ and $f \in X$. Then $B \in \mathcal{L}(X)$. Indeed, we have, for every $h \in \mathbb{N}$ and $f \in X$, that

$$
p_{h}(B f)=\sup _{x \in[0, h]}|x B f(x)|=\sup _{x \in[0, h]}\left|x^{2} f(x)\right| \leq h p_{h}(f) .
$$


Moreover, $(B, X)$ is dissipative. To see this, we observe, for every $f \in X$ and $x, \lambda>0$, that

$$
x|\lambda f(x)-B f(x)|=x|\lambda f(x)+x f(x)|=x(\lambda+x)|f(x)| \geq \lambda x|f(x)|
$$

and hence, it follows, for every $h \in \mathbb{N}$, that

$$
p_{h}((\lambda I-B) f) \geq \lambda p_{h}(f) .
$$

We claim that the operator $(A+B, D(A))$ generates an equicontinuous $C_{0}$-semigroup on $X$. To see this, it suffices via Proposition 4.5 to show that $\operatorname{rg}(I-(A+B))=X$. So, fix $g \in X$ and consider the equation $f-(A+B) f=g$ on $] 0,+\infty$ [, i.e., $x f^{\prime}(x)+(2+x) f(x)=g(x)$, for $x>0$. As it is easy to calculate, the general solution $f$ is given by

$$
f(x):=\frac{1}{x^{2} e^{x}} \int_{0}^{x} y e^{y} g(y) d y+\frac{c}{x^{2} e^{x}}, \quad x>0, c \in \mathbb{C} .
$$

Since $g \in X$, similar arguments to the previous ones yield that the function $f$ given in (4.8) belongs to $D(A)$ whenever $c=0$. So, $(4.4)$ is satisfied, i.e., $\operatorname{rg}(I-(A+B))=X$.

Remark 4.7. (i) In case the condition $\overline{\operatorname{rg}(\lambda I-(A+B))}=X$ is satisfied for some $\lambda>0$ instead of the identity in (4.4), the arguments of the proof of Proposition 4.5 yield that the closure of $(A+B, D(A))$ generates a $\Gamma$-contractively equicontinuous $C_{0}$-semigroup on $X$.

(ii) If $\overline{\operatorname{rg}(I-B R(\lambda, A))}=X$, for some $\lambda>0$, then also $\overline{\operatorname{rg}(\lambda I-(A+B))}=X$. Indeed, from the identity

it follows that

$$
(\lambda I-(A+B)) x=(I-B R(\lambda, A))(\lambda I-A) x, \quad x \in D(A),
$$

$$
(\lambda I-(A+B))(D(A))=(I-B R(\lambda, A))(\lambda I-A)(D(A))=(I-B R(\lambda, A))(X) .
$$

Hence, we have

$$
\overline{\operatorname{rg}(\lambda I-(A+B))}=\overline{\operatorname{rg}(I-B R(\lambda, A))}=X .
$$

Next, we introduce the notion of $(\Gamma, A)$-bounded operators and show some related properties.

Definition 4.8. Let $X$ be a lcHs and let $\Gamma \subseteq \Gamma_{X}$ determine the lc-topology of $X$. Let $(A, D(A))$ and $(B, D(B))$ be two operators on $X$. The operator $(B, D(B))$ is called $(\Gamma, A)$-bounded if $D(A) \subseteq D(B)$ and

$$
\forall p \in \Gamma, \exists a_{p}, b_{p}>0, \forall x \in D(A), p(B x) \leq a_{p} p(A x)+b_{p} p(x) .
$$

For each $p \in \Gamma$, the number $a_{p, 0}:=\inf \left\{a_{p}>0:(4.10)\right.$ holds for some $\left.b_{p}\right\}$ is called $(p, A)$-bound of $B$.

As in the setting of Banach spaces, the following result holds.

Proposition 4.9. Let $(A, D(A))$ be a closed operator on a complete lcHs $X$. Let $(B, D(B))$ be a $(\Gamma, A)$-bounded operator on $X$ for some $\Gamma \subseteq \Gamma_{X}$ with $\Gamma$ generating the lc-topology of $X$. If the $(p, A)$ bound $a_{p, 0}<1$ for all $p \in \Gamma$, then $(A+B, D(A))$ is closed.

Proof. Fix $p \in \Gamma$. Then there exists $a_{p}<1$ such that the inequality in (4.10) is satisfied. So we obtain, for every $x \in D(A)$, that

$$
\begin{aligned}
p(A x) & =p((A+B) x-B x) \leq p((A+B) x)+p(B x) \\
& \leq p((A+B) x)+a_{p} p(A x)+b_{p} p(x),
\end{aligned}
$$

and hence, that $\left(1-a_{p}\right) p(A x) \leq p((A+B) x)+b_{p} p(x)$. We also obtain, for every $x \in D(A)$, that

$$
p((A+B) x) \leq p(A x)+p(B x) \leq\left(1+a_{p}\right) p(A x)+b_{p} p(x) .
$$

Therefore, it follows, for every $x \in D(A)$, that

$$
\left(1-a_{p}\right) p(A x)+b_{p} p(x) \leq p((A+B) x)+2 b_{p} p(x) \leq\left(1+a_{p}\right) p(A x)+3 b_{p} p(x) .
$$

Now, we fix $\left(x_{\alpha}\right)_{\alpha} \subseteq D(A)$ such that $x_{\alpha} \rightarrow x$ and $(A+B) x_{\alpha} \rightarrow y$ in $X$. By the left-hand side inequality in (4.11), we have, for every $p \in \Gamma$ and $\alpha, \alpha^{\prime}$, that

$$
p\left(A\left(x_{\alpha}-x_{\alpha^{\prime}}\right)\right) \leq \frac{1}{1-a_{p}} p\left((A+B)\left(x_{\alpha}-x_{\alpha^{\prime}}\right)\right)+\frac{2 b_{p}}{1-a_{p}} p\left(x_{\alpha}-x_{\alpha^{\prime}}\right) .
$$


Consequently, $\left(A x_{\alpha}\right)_{\alpha}$ is a Cauchy net in $X$. Since $X$ is complete, there exists $A x_{\alpha} \rightarrow z$ in $X$. But, $(A, D(A))$ is closed and hence, $x \in D(A)$ and $A x=z$.

Finally, by the right-hand side inequality in (4.11) we obtain, for every $p \in \Gamma$ and $\alpha$, that

$$
p\left((A+B)\left(x-x_{\alpha}\right)\right) \leq\left(1+a_{p}\right) p\left(A\left(x-x_{\alpha}\right)\right)+3 b_{p} p\left(x-x_{\alpha}\right) .
$$

Consequently, $(A+B) x-(A+B) x_{\alpha} \rightarrow 0$ in $X$. But, also $(A+B) x-(A+B) x_{\alpha} \rightarrow(A+B) x-y$ in $X$. Then, we conclude that $(A+B) x=y$. Accordingly, $(A+B, D(A))$ is closed.

We recall that if $(A, D(A))$ and $(B, D(B))$ are two operators on a Banach space $(X,\|\|)$ satisfying the first part of the assumptions in Proposition 4.5 but nothing is assumed about the identity in (4.4), then the operator $(A+B, D(A))$ surely generates a contractive $C_{0}$-semigroup on $X$ whenever the operator $B$ is $(\|\|, A)$-bounded with bound $a_{\|\|, 0}<1$; see , f.i., [13, Theorem III.2.7]. In what follows, we extend the result in the setting of some classes of Fréchet spaces.

Theorem 4.10. Let $X=\cap_{j \in \mathbb{N}} X_{j}$ be a Fréchet space which is the intersection of a reduced sequence of Banach spaces $\left\{\left(X_{j}, p_{j}\right)\right\}_{j \in \mathbb{N}}$ satisfying $X_{j+1} \subseteq X_{j}$ with $p_{j}(x) \leq p_{j+1}(x)$ for each $j \in \mathbb{N}$ and $x \in X_{j+1}$ and let $\Gamma=\left\{p_{j}\right\}_{j \in \mathbb{N}}$.

Let $(A, D(A))$ be the infinitesimal generator of a $\Gamma$-contractively equicontinuous $C_{0}$-semigroup on $X$. Let $(B, D(B))$ be a $\Gamma$-dissipative operator on $X$. If the operator $(B, D(B))$ is $(\Gamma, A)$-bounded with $a_{p_{j}, 0}<1$ for all $j \in \mathbb{N}$, then $(A+B, D(A))$ generates a $\Gamma$-contractively equicontinuous $C_{0}$-semigroup on $X$.

Proof. The argument for the proof of Proposition 4.5 shows that $(A+B, D(A))$ is a $\Gamma$-dissipative, closed densely defined operator on $X$. Since $X$ is complete, to conclude the proof it suffices to see that $\operatorname{rg}(\lambda I-(A+B))=X$ for some $\lambda>0$; see Theorem 3.13.

Now, fix $j \in \mathbb{N}$. Since $X \subseteq X_{j}$ continuously and $\bar{X}=X_{j}$, from $p_{j}(T(t) x) \leq p_{j}(x)$, for $t \geq 0$ and $x \in X$, it follows that each $T(t)$, for $t>0$, admits a continuous linear extension $T_{j}(t)$ on $X_{j}$ satisfying $p_{j}\left(T_{j}(t) x\right) \leq p_{j}(x)$, for $t \geq 0$ and $x \in X_{j}$. In particular, $\left(T_{j}(t)\right)_{t \geq 0}$ is a $C_{0}$-semigroup on $X_{j}$. Indeed, the semigroup law is clearly satisfied. On the other hand, $T_{j}(t) x \rightarrow x$ as $t \rightarrow 0^{+}$for all $x \in X_{j}$. To see this, take a sequence of positive numbers $t_{k} \rightarrow 0$. Since the family $\left(T_{j}\left(t_{k}\right)\right)_{k \in \mathbb{N}}$ is equicontinuous in $X_{j}$ and converges on the dense set $X$, the conclusion follows by a straight forward argument.

Let $\left(A_{j}, D\left(A_{j}\right)\right)$ denote the infinitesimal generator of $\left(T_{j}(t)\right)_{t \geq 0}$. We show that $\left(A_{j}, D\left(A_{j}\right)\right)$ is the closure of $(A, D(A))$ on $X_{j}$. To see this, we first observe that $\left.R\left(\lambda, A_{j}\right)\right|_{X}=R(\lambda, A)$ for each $\lambda>0$. This fact follows from $\left.T_{j}(t)\right|_{X}=T(t)$, for all $t \geq 0$, and from $X \subseteq X_{j}$ continuously. Indeed, we have, for every $\lambda>0$ and $x \in X$, that

$$
R(\lambda, A) x=\int_{0}^{\infty} T(t) x d t=\int_{0}^{\infty} T_{j}(t) x d t=R\left(\lambda, A_{j}\right) x .
$$

Consequently, $D(A)=R(1, A)(X)=R\left(1, A_{j}\right)(X) \subseteq D\left(A_{j}\right)$. The property $\left.T_{j}(t)\right|_{X}=T(t)$, for all $t \geq 0$, also implies, for every $x \in D(A)$, that $A x=\lim _{t \rightarrow 0^{+}} \frac{T(t) x-x}{t}=\lim _{t \rightarrow 0^{+}} \frac{T_{j}(t) x-x}{t}=A_{j} x$, i.e., $\left.A_{j}\right|_{D(A)}=A$. Therefore, $\left(A_{j}, D\left(A_{j}\right)\right)$ is a closed extension of $(A, D(A))$ on $X_{j}$ and hence, $(A, D(A))$ is closable on $X_{j}$. But, if $x \in D\left(A_{j}\right)$, there exists $y \in X_{j}$ such that $x=R\left(1, A_{j}\right) y$. Since $D(A)$ is dense in $X$ and so in $X_{j}$, there exists $\left(y_{h}\right)_{h \in \mathbb{N}} \subseteq D(A)$ satisfying $y_{h} \rightarrow y$ in $X_{j}$ for $h \rightarrow \infty$. For each $h \in \mathbb{N}$, let $x_{h}:=R(1, A) y_{h}$. Then $\left(x_{h}\right)_{h \in \mathbb{N}} \subseteq D(A)$ and $x_{h}=R(1, A) y_{h}=R\left(1, A_{j}\right) y_{h} \rightarrow R\left(1, A_{j}\right) y=x$ in $X_{j}$ for $h \rightarrow \infty$. Also, $A x_{h}=A R(1, A) y_{h}=R(1, A) y_{h}-y_{h} \rightarrow x-y=R\left(1, A_{j}\right) y-y=A_{j} R\left(1, A_{j}\right) y=A_{j} x$ in $X_{j}$ for $h \rightarrow \infty$. Since $x \in D\left(A_{j}\right)$ is arbitrary, by Proposition 3.4(ii) we can conclude that $\left(A_{j}, D\left(A_{j}\right)\right)$ is the closure of $(A, D(A))$ on $X_{j}$.

Next, we show that also $B$ extends to a linear operator on $D\left(A_{j}\right)$ which is $\left(p_{j}, A_{j}\right)$-bounded with $a_{p_{j}, 0}<1$, i.e., there exists an operator $B_{j}: D\left(A_{j}\right) \subseteq X_{j} \rightarrow X_{j}$ satisfying $\left.B_{j}\right|_{D(A)}=B$ and

$$
p_{j}\left(B_{j} x\right) \leq a_{p_{j}} p_{j}\left(A_{j} x\right)+b_{p_{j}} p_{j}(x), \quad x \in D\left(A_{j}\right),
$$

for some positive constants $a_{p_{j}}$ and $b_{p_{j}}$, with $a_{p_{j}, 0}=\inf \left\{a_{p_{j}}:(4.12)\right.$ holds for some $\left.b_{p_{j}}>0\right\}<1$. To see this, fix $x \in D\left(A_{j}\right)$. Since $\left(A_{j}, D\left(A_{j}\right)\right)$ is the closure of $(A, D(A))$ in $X_{j}$, via Proposition 3.4(ii) 
we can find a sequence $\left(x_{h}\right)_{h \in \mathbb{N}} \subseteq D(A)$ such that $x_{h} \rightarrow x$ and $A x_{h} \rightarrow A_{j} x$ in $X_{j}$ for $h \rightarrow \infty$. But, by (4.10) we have

$$
p_{j}\left(B\left(x_{h}-x_{k}\right)\right) \leq a_{p_{j}} p_{j}\left(A\left(x_{h}-x_{k}\right)\right)+b_{p_{j}} p_{j}\left(x_{h}-x_{k}\right), \quad h, k \in \mathbb{N},
$$

for some positive constants $a_{p_{j}}$ and $b_{p_{j}}$. Accordingly, $\left(B x_{h}\right)_{h \in \mathbb{N}}$ is a Cauchy sequence in $X_{j}$ and hence, it converges in $X_{j}$, say to $B_{j} x$, i.e., $B_{j} x:=\lim _{h \rightarrow \infty} B x_{h}$. Clearly, the operator $\left(B_{j}, D\left(A_{j}\right)\right)$ is linear. Moreover, again by (4.10) we have

$$
p_{j}\left(B x_{h}\right) \leq a_{p_{j}} p_{j}\left(A x_{h}\right)+b_{p_{j}} p_{j}\left(x_{h}\right), \quad h \in \mathbb{N},
$$

for some positive constants $a_{p_{j}}$ and $b_{p_{j}}$. Letting $h \rightarrow \infty$ and keeping in mind that $p_{j}$ is continuous on $X_{j}$, it follows that

$$
p_{j}\left(B_{j} x\right) \leq a_{p_{j}} p_{j}\left(A_{j} x\right)+b_{p_{j}} p_{j}(x) .
$$

Since $x \in D\left(A_{j}\right)$ is arbitrary, the inequality (4.12) necessarily holds. The fact that $a_{p_{j}, 0}<1$ follows by (4.12) and the assumptions.

The operator $\left(B_{j}, D\left(A_{j}\right)\right)$ is also $p_{j}$-dissipative. Indeed, by assumption we have

$$
p_{j}((\lambda I-B) x) \geq \lambda p_{j}(x), \quad \lambda>0, x \in D(A) .
$$

Now, fixed $x \in D\left(A_{j}\right)$ and repeating the argument above, we can find a sequence $\left(x_{h}\right)_{h \in \mathbb{N}} \subseteq D(A)$ such that $x_{h} \rightarrow x, A x_{h} \rightarrow A_{j} x$ and $B x_{h} \rightarrow B_{j} x$ in $X_{j}$ for $h \rightarrow \infty$. Applying the above inequality we obtain

$$
p_{j}\left((\lambda I-B) x_{h}\right) \geq \lambda p_{j}\left(x_{h}\right), \quad \lambda>0, h \in \mathbb{N},
$$

and so, letting $h \rightarrow \infty$, it follows that

$$
p_{j}\left(\left(\lambda I-B_{j}\right) x\right) \geq \lambda p_{j}(x), \quad \lambda>0 .
$$

The arbitrariness of $x$ in $D\left(A_{j}\right)$ then ensures that $\left(B_{j}, D\left(A_{j}\right)\right)$ is $p_{j}$-dissipative.

As $\left(A_{j}, D\left(A_{j}\right)\right)$ is the infinitesimal generator of a $p_{j}$-contractive $C_{0}$-semigroup on $X_{j}$ and $\left(B_{j}, D\left(A_{j}\right)\right)$ is a $p_{j}$-dissipative, $\left(p_{j}, A_{j}\right)$-bounded operator with $a_{p_{j}, 0}<1$, the operator $\left(A_{j}+B_{j}, D\left(A_{j}\right)\right)$ generates a $p_{j}$-contractive $C_{0}$-semigroup $\left(S_{j}(t)\right)_{t \geq 0}$ on $X_{j}$; see, f.i., [13, Theorem III.2.7]. Accordingly, $(0, \infty) \subseteq$ $\rho\left(A_{j}+B_{j}\right)$.

The above construction yields, for every $j \in \mathbb{N}$, that $\left(A_{j}, D\left(A_{j}\right)\right)$ is also the closure of the operator $\left(A_{j+1}, D\left(A_{j+1}\right)\right)$ on $X_{j}$ and that $\left.B_{j}\right|_{D\left(A_{j+1}\right)}=B_{j+1}$ and $\left.B_{j}\right|_{D(A)}=B$. We will see that both facts imply that $D(A)=\cap_{j \in \mathbb{N}} D\left(A_{j}\right)$ and that $(0, \infty) \subseteq \rho(A+B)$.

We first show that $D(A)=\cap_{j \in \mathbb{N}} D\left(A_{j}\right)$. Set $D(\bar{A}):=\cap_{j \in \mathbb{N}} D\left(A_{j}\right)$ and define $\bar{A} x:=A_{1} x$ for $x \in D(\bar{A})$. Clearly, $(\bar{A}, D(\bar{A}))$ is a well defined operator on $X$ and also an extension of $(A, D(A))$ on $X$. In particular, $(\bar{A}, D(\bar{A}))$ is a closed operator on $X$. Indeed, if $\left(x_{h}\right)_{h \in \mathbb{N}} \subseteq D(\bar{A})$ satisfies $x_{h} \rightarrow x$ and $\bar{A} x_{h} \rightarrow y$ in $X$ for $h \rightarrow \infty$, then, for every $j \in \mathbb{N},\left(x_{h}\right)_{h \in \mathbb{N}} \subseteq D\left(A_{j}\right)$ and $x_{h} \rightarrow x, A_{j} x_{h}=\bar{A} x_{h} \rightarrow y$ in $X_{j}$ as $h \rightarrow \infty$. Since $\left(A_{j}, D\left(A_{j}\right)\right)$ is a closed operator on $X_{j}$ for all $j \in \mathbb{N}$, it follows that $x \in D\left(A_{j}\right)$ and $A_{j} x=y$ for all $j \in \mathbb{N}$. Accordingly, $x \in \cap_{j \in \mathbb{N}} D\left(A_{j}\right)=D(\bar{A})$ and $\bar{A} x=A_{1} x=y$. So, $(\bar{A}, D(\bar{A}))$ is a closed operator on $X$. Actually, $(\bar{A}, D(\bar{A}))$ is the closure of $(A, D(A))$ on $X$. In fact, fixed $x \in D(\bar{A})$, for every $j \in \mathbb{N}$ there exists $x_{j} \in D(A)$ such that $p_{j}\left(x-x_{j}\right)<1 / j$ and $p_{j}\left(\bar{A} x-A x_{j}\right)=p_{j}\left(A_{j} x-A x_{j}\right)<1 / j$ (see Proposition 3.4(ii) and keep in mind the construction of $\left(A_{j}, D\left(A_{j}\right)\right)$ ). It follows that $x_{j} \rightarrow x$ and $A x_{j} \rightarrow \bar{A} x$ in $X$ for $j \rightarrow \infty$. By Proposition 3.4(ii) this shows that $(\bar{A}, D(\bar{A}))$ is indeed the closure of $(A, D(A))$. But, $(A, D(A))$ is closed and then $(A, D(A))=(\bar{A}, D(\bar{A}))$.

We now show that $(0, \infty) \subseteq \rho(A+B)$. To this end, fix $\lambda>0$ and $y \in X$. Then, for every $j \in \mathbb{N}$ there exists $x_{j} \in D\left(A_{j}\right)$ such that $\left(\lambda I-\left(A_{j}+B_{j}\right)\right) x_{j}=y$. But, $\left.\left(A_{j}+B_{j}\right)\right|_{D\left(A_{j+1}\right)}=A_{j+1}+B_{j+1}$ for $j \in \mathbb{N}$ and hence, $\left(\lambda I-\left(A_{j}+B_{j}\right)\right) x_{j}=y=\left(\lambda I-\left(A_{j}+B_{j}\right)\right) x_{j+1}$ for $j \in \mathbb{N}$. As $\left(\lambda I-\left(A_{j}+B_{j}\right)\right)$ is injective, it follows that $x_{j}=x_{j+1}$ for all $j \in \mathbb{N}$. So, if we set $x:=x_{1}$, then we have that $x_{j}=x$ for all $j \in \mathbb{N}$. Consequently, $x \in \cap_{j \in \mathbb{N}} D\left(A_{j}\right)$, i.e., $x \in D(A)$ (by the proof above), and hence, $(\lambda I-(A+B)) x=\left(\lambda I-\left(A_{1}+B_{1}\right)\right) x_{1}=y$. This shows that $(\lambda I-(A+B))$ is a surjective operator. So, the proof is complete.

The space $C^{\infty}([0,1])=\cap_{j \in \mathbb{N}} C^{j}([0,1])$ is a classical example of a Fréchet function space satisfying the assumptions in Theorem 4.10. The next example is more involved. 
Example 4.11. Let $1<p \leq \infty$ and $L^{p-}:=\cap_{1<r<p} L^{r}([0,1])$. Then $L^{p-}$ is a reflexive Fréchet space with respect the lc-topology generated by the norms $\|f\|_{h}:=\left(\int_{0}^{1}|f(x)|^{r_{h}} d x\right)^{1 / r_{h}}$, for $h \in \mathbb{N}$ and $f \in L^{p-}$, where $\left(r_{h}\right)_{h}$ is an increasing sequence of real numbers satisfying $1<r_{h} \uparrow p$ for $h \rightarrow \infty$. Let $\Gamma:=\left\{\|\|_{h}\right\}_{h \in \mathbb{N}}$.

We consider the operator $A f:=f^{\prime \prime}$ with domain $D(A):=W^{2, p-} \cap W_{0}^{1, p-}$, where $W^{2, p-}:=$ $\cap_{1<r<p} W^{2, r}([0,1])$ and $W_{0}^{1, p-}:=\cap_{1<r<p} W_{0}^{1, r}([0,1])$. Then $(A, D(A))$ is a $\Gamma$-dissipative operator in $L^{p-}$ with $\operatorname{rg}(\lambda I-A)=L^{p-}$ for all $\lambda>0$. Indeed, fixed $h \in \mathbb{N}$ and $\lambda>0$, we have that $\|(\lambda I+A) f\|_{h} \geq\|f\|_{h}$ for all $f \in W^{2, r_{h}}([0,1]) \cap W_{0}^{1, r_{h}}([0,1])$ (see, f.i., $[25$, Theorem 3.6]) and hence, for all $f \in D(A)$. Since $h \in \mathbb{N}$ and $\lambda>0$ are arbitrary, the $\Gamma$-dissipativity of $(A, D(A))$ follows.

Now, fix $\lambda>0$ and recall that the operator $\lambda I-A: W^{2, r_{h}}([0,1]) \cap W_{0}^{1, r_{h}}([0,1]) \rightarrow L^{r_{h}}([0,1])$ is bijective for every $h \in \mathbb{N}$; see, again, [25, Theorem 3.6]. This implies that also the operator $\lambda I-A: W^{2, p-} \cap W_{0}^{1, p-} \rightarrow L^{p-}$ is bijective. To see this, it is enough to proceed as in the proof of Theorem 4.10. Indeed, fixed $g \in L^{p-}$, we have that $g \in L^{r_{h}}([0,1])$ for all $h \in \mathbb{N}$ and hence, there exists $f_{h} \in W^{2, r_{h}}([0,1]) \cap W_{0}^{1, r_{h}}([0,1])$ such that $(\lambda-A) f_{h}=g$. It follows that $(\lambda-A) f_{h}=g=(\lambda-A) f_{h+1}$ for all $h \in \mathbb{N}$. But, for every $h \in \mathbb{N}, \lambda I-A$ is an injective operator in $L^{r_{h}}([0,1])$ and $f_{h+1}, f_{h} \in$ $W^{2, r_{h}}([0,1]) \cap W_{0}^{1, r_{h}}([0,1])$. So, we have $f_{h}=f_{h+1}$ for all $h \in \mathbb{N}$. If we set $f:=f_{1}$, we then obtain that $f \in D(A)$ and $(\lambda I-A) f=(\lambda I-A) f_{1}=g$. This shows the surjectivity of the operator $\lambda I-A$ in $L^{p-}$.

Since the operator $(A, D(A))$ is also densely defined in $L^{p-}$, we can conclude via Theorem 3.13 that it generates a $\Gamma$-contractively equicontinuous $C_{0}$-semigroup in $L^{p-}$.

Next, we consider the operator $B f:=-f^{\prime}$ with domain $D(A)$. Then $(B, D(A))$ is a $\Gamma$-dissipative operator on $L^{p-}$. To see this, we fix $f \in D(A) \backslash\{0\}$ and $h \in \mathbb{N}$. As in Example 4.1 one shows that the linear functional $F$ defined via the function $g(x):=\overline{f(x)}|f(x)|^{r_{h}-2}\|f\|_{h}^{1-r_{h}}$ if $f(x) \neq 0$ and $g(x):=0$ otherwise belongs to $d\|\|_{h}$. Now, we write $f=\alpha+i \beta$ and, hence, $\operatorname{Re}\left(f^{\prime} \bar{f}\right)(x)=\operatorname{Re}\left(f \bar{f}^{\prime}\right)(x)=$ $\alpha(x) \alpha^{\prime}(x)+\beta(x) \beta^{\prime}(x)=\frac{1}{2}(f \bar{f})^{\prime}(x)$ for all $x \in[0,1]$. Then, we have

$$
\begin{aligned}
\operatorname{Re}\langle B f, F\rangle & =-\|f\|_{h}^{1-r_{h}} \operatorname{Re} \int_{0}^{1} f^{\prime}(x) \overline{f(x)}|f(x)|^{r_{h}-2} d x \\
& =-\|f\|_{h}^{1-r_{h}} \int_{0}^{1}\left(\alpha^{2}(x)+\beta^{2}(x)\right)^{\frac{r_{h}-2}{2}}\left(\alpha(x) \alpha^{\prime}(x)+\beta(x) \beta^{\prime}(x)\right) d x \\
& =-\|f\|_{h}^{1-r_{h}}\left[\frac{1}{r_{h}}|f(x)|^{r_{h}}\right]_{0}^{1}=0 .
\end{aligned}
$$

On the other hand, the operator $(B, D(A))$ is $(\Gamma, A)$-bounded with bound $a_{\|\|_{h}, 0}=0<1$ for all $h \in \mathbb{N}$. Indeed, we have, for every $h \in \mathbb{N}$ and $\varepsilon>0$, that

$$
\|B f\|_{h} \leq \varepsilon\|A f\|_{h}+\frac{9}{\varepsilon}\|f\|_{h}, \quad f \in D(A)
$$

see, f.i., [13, Chap.III, §2, Example 2.2].

Since the space $L^{p-}$ belongs to the class of Fréchet spaces satisfying the assumptions of Theorem 4.10 , we can now conclude that the operator $(A+B, D(A))$ generates a $\Gamma$-contractively equicontinuous $C_{0}$-semigroup $(T(t))_{t \geq 0}$ on $L^{p-}$.

For each $h \in \mathbb{N}$ let $s_{h}>1$ ( $q>1$, resp.) denote the conjugate exponent of $r_{h}$ (of $p$, resp.), i.e., $\frac{1}{r_{h}}+\frac{1}{s_{h}}=1$. We observe that the dual semigroup $\left(T^{\prime}(t)\right)_{t \geq 0}$ acting on the dual space $\left(L^{p-}\right)^{\prime}=$ $\cup_{h=1}^{\infty}\left(L^{r_{h}}[0,1]\right)^{\prime}=\cup_{h=1}^{\infty} L^{s_{h}}[0,1]=: L^{q+}$ is clearly $\sigma\left(\left(L^{p-}\right)^{\prime}, L^{p-}\right)$-continuous on $\left(L^{p-}\right)^{\prime}$. On the other hand, as it is routine to verify, $\left(T^{\prime}(t)\right)_{t \geq 0}$ is equicontinuous on the strong dual $\left(L^{p-}\right)_{\beta}^{\prime}$ of $L^{p-}$. Consequently, as the Fréchet space $L^{p-}$ is reflexive and its strong dual $\left(L^{p-}\right)_{\beta}^{\prime}$ is complete (and barrelled), the dual semigroup $\left(T^{\prime}(t)\right)_{t \geq 0}$ is also $C_{0}$-continuous on $\left(L^{p-}\right)_{\beta}^{\prime}$; see [4, Proposition 1]. In particular, an argument of duality shows that its infinitesimal generator $(C, D(C))$ is given by

$$
C g=g^{\prime \prime}+g^{\prime}, \quad g \in \cup_{h=1}^{\infty} W^{2, s_{h}}([0,1]) \cap W_{0}^{1, s_{h}}([0,1]) .
$$


Our next purpose is to prove that Theorem 4.10 continues to hold also for another class of Fréchet spaces, the so-called quojections. First, some preliminaries are required.

A Fréchet space $X$ is always a projective limit of continuous linear operators $S_{j}: X_{j+1} \rightarrow X_{j}$, for $j \in \mathbb{N}$, each $X_{j}$ being a Banach space. If it is possible to choose $X_{j}$ and $S_{j}$ such that each $S_{j}$ is surjective and $X$ is isomorphic to the projective limit $\operatorname{proj}_{j}\left(X_{j}, S_{j}\right)$, then $X$ is called a quojection, [10, Section 5]. Banach spaces and countable products of Banach spaces are quojections. Actually, every quojection is the quotient of a countable product of Banach spaces, [11]. In [24], Moscatelli gave the first examples of quojections which are not isomorphic to countable products of Banach spaces. Concrete examples of quojections are $\omega=\mathbb{C}^{\mathbb{N}}$, the spaces $L_{l o c}^{p}(\Omega)$, with $1 \leq p \leq \infty$, and $C^{(m)}(\Omega)$, for all $m \in \mathbb{N}_{0}$. The space $X$ given in Example 4.6 is a quojection also. Indeed, the above function spaces are isomorphic to countable products of Banach spaces. Moreover, the spaces of continuous functions $C(\Lambda)$, with $\Lambda$ a $\sigma$-compact completely regular topological space, endowed with the compact open topology are also examples of quojections. For further information on quojections we refer to the survey paper [22] and the references therein.

Let $X$ be a quojection Fréchet space, i.e., $X=\operatorname{proj}_{j}\left(X_{j}, Q_{j, j+1}\right)$ with $Q_{j, j+1}: X_{j+1} \rightarrow X_{j}$ surjective for all $j \in \mathbb{N}$, and $p_{j}$ a norm of $X_{j}$ for all $j \in \mathbb{N}$. Then there exist continuous, surjective linking maps $Q_{j}: X \rightarrow X_{j}$ such that

$$
Q_{j, j+1} \circ Q_{j+1}=Q_{j}, \quad j \in \mathbb{N} .
$$

In particular, if we set $q_{j}:=p_{j} \circ Q_{j}$ for all $j \in \mathbb{N}$, then $\left\{q_{j}\right\}_{j \in \mathbb{N}} \subseteq \Gamma_{X}$ generates the lc-topology of $X$.

Theorem 4.12. Let $X=\operatorname{proj}_{j}\left(X_{j}, Q_{j, j+1}\right)$ be a quojection Fréchet space and let $q_{j}:=p_{j} \circ Q_{j}$ with $p_{j}$ a norm on $X_{j}$ and $Q_{j}$ satisfying the identity in (4.13) for all $j \in \mathbb{N}$. Let $\Gamma:=\left\{q_{j}\right\}_{j \in \mathbb{N}}$. Let $(A, D(A))$ be the infinitesimal generator of a $\Gamma$-contractively equicontinuous $C_{0}$-semigroup $(T(t))_{t \geq 0}$ on $X$ and $(B, D(B))$ be a $\Gamma$-dissipative operator on $X$. If the operator $(B, D(B))$ is $(\Gamma, A)$-bounded with $a_{q_{j}, 0}<1$ for all $j \in \mathbb{N}$, then $(A+B, D(A))$ generates a $\Gamma$-contractively equicontinuous $C_{0}$-semigroup on $X$.

Proof. The argument for the proof of Proposition 4.5 shows that $(A+B, D(A))$ is a $\Gamma$-dissipative, closed densely defined operator on $X$. Since $X$ is complete, to conclude the proof it then suffices to show that $\operatorname{rg}(\lambda I-(A+B))=X$ for some $\lambda>0$; see Theorem 3.13. To this end, we first observe that $(0, \infty) \subseteq \rho(A)$ and that

$$
q_{j}(R(\lambda, A) x) \leq \frac{1}{\lambda} q_{j}(x), \quad \lambda>0, j \in \mathbb{N}, x \in X,
$$

as the semigroup $(T(t))_{t \geq 0}$ is $\Gamma$-contractively equicontinuous.

Fix $j \in \mathbb{N}$. Define a family $\left(T_{j}(t)\right)_{t \geq 0}$ of operators on the Banach space $X_{j}$ via

$$
T_{j}(t) Q_{j} x:=Q_{j} T(t) x, \quad x \in X, t \geq 0 .
$$

Proceeding as in the proof of Theorem 3.3 of [3] one shows via (4.15) that each $T_{j}(t)$, for $t \geq 0$, is a well defined linear operator on $X_{j}$ (with $T_{j}(0)=I$ on $X_{j}$ ) and that the semigroup law is satisfied. Moreover, via (4.15) we obtain that

$$
p_{j}\left(T_{j}(t) \hat{x}\right)=p_{j}\left(T_{j}(t) Q_{j} x\right)=p_{j}\left(Q_{j} T(t) x\right)=q_{j}(T(t) x) \leq q_{j}(x)=p_{j}\left(Q_{j} x\right)
$$

for all $\hat{x} \in X_{j}$ and $x \in X$ with $Q_{j} x=\hat{x}$. It follows that

$$
p_{j}\left(T_{j}(t) \hat{x}\right) \leq p_{j}(\hat{x}), \quad \hat{x} \in X_{j} .
$$

Since $p_{j}$ is the norm of $X_{j}$, (4.16) ensures the continuity of $T_{j}(t)$, for all $t \geq 0$, and that $\left(T_{j}(t)\right)_{t \geq 0} \subseteq$ $\mathcal{L}\left(X_{j}\right)$ is a $p_{j}$-contraction semigroup on $X_{j}$. Actually, $\left(T_{j}(t)\right)_{t \geq 0}$ is a $C_{0}$-semigroup on the Banach space $X_{j}$. Indeed, for every $x \in X$, we have $\lim _{t \rightarrow 0^{+}} T_{j}(t) Q_{j} x=\lim _{t \rightarrow 0^{+}} Q_{j} T(t) x=Q_{j} x$ via (4.15) and the continuity of $Q_{j}$. Since $Q_{j}$ is surjective, it follows that $\left(T_{j}(t)\right)_{t \geq 0}$ is strongly continuous on $X_{j}$.

If we denote by $\left(A_{j}, D\left(A_{j}\right)\right)$ the infinitesimal generator of $\left(T_{j}(t)\right)_{t \geq 0}$, then from (4.15) it follows that

$$
\lim _{t \rightarrow 0^{+}} \frac{T_{j}(t) Q_{j} x-Q_{j} x}{t}=\lim _{t \rightarrow 0^{+}} Q_{j}\left(\frac{T(t) x-x}{t}\right)=Q_{j} A x, \quad x \in D(A) .
$$


Accordingly, $Q_{j}(D(A)) \subseteq D\left(A_{j}\right)$ and

$$
A_{j} Q_{j} x=Q_{j} A x, \quad x \in D(A) .
$$

Actually, $D\left(A_{j}\right)=Q_{j}(D(A))$. To show this, we observe that from (4.15) we also obtain that

$$
\begin{aligned}
R\left(\lambda, A_{j}\right) Q_{j} x & =\int_{0}^{\infty} e^{-\lambda t} T_{j}(t) Q_{j} x d t=\int_{0}^{\infty} e^{-\lambda t} Q_{j} T(t) x d t \\
& =Q_{j}\left(\int_{0}^{\infty} e^{-\lambda t} T(t) x d t\right)=Q_{j} R(\lambda, A) x, \quad x \in X, \lambda>0,
\end{aligned}
$$

where the third equality in (4.18) easily follows by approximation with Riemann sums and the continuity of $Q_{j}$. Fixed any $\lambda>0$, if $\hat{y} \in D\left(A_{j}\right)$, there exists $\hat{x} \in X_{j}$ such that $R\left(\lambda, A_{j}\right) \hat{x}=\hat{y}$. Since $\hat{x}=Q_{j} x$ for some $x \in X$, it follows from (4.18) that $\hat{y}=R\left(\lambda, A_{j}\right) \hat{x}=R\left(\lambda, A_{j}\right) Q_{j} x=Q_{j} R(\lambda, A) x$. As $R(\lambda, A) x \in D(A)$ and so $Q_{j} R(\lambda, A) x \in Q_{j}(D(A))$, we have $\hat{y} \in Q_{j}(D(A))$.

Next, define an operator $B_{j}$ on $D\left(A_{j}\right)$ as

$$
B_{j} Q_{j} x:=Q_{j} B x, \quad x \in D(A) .
$$

The operator $B_{j}: D\left(A_{j}\right)=Q_{j}(D(A)) \subseteq X_{j} \rightarrow X_{j}$ is well defined and linear. Indeed, if $Q_{j} x=Q_{j} y$ for some $x, y \in D(A)$, then $Q_{j}(x-y)=0$, i.e., $x-y \in \operatorname{Ker} Q_{j}=\operatorname{Ker} q_{j}$ and hence, $q_{j}(x-y)=0$. Also $q_{j}(A(x-y))=0$. Indeed, from $Q_{j}(x-y)=0$ and (4.17) we obtain that $Q_{j} A(x-y)=A_{j} Q_{j}(x-y)=0$ and so $q_{j}(A(x-y))=0$ (by repeating the argument above). Since $(B, D(B))$ is $(\Gamma, A)$-bounded, we can now apply (4.10) with $p=q_{j}$ and $x-y \in D(A)$, thereby obtaining that

$$
q_{j}(B(x-y)) \leq a_{q_{j}} q_{j}(A(x-y))+b_{q_{j}} q_{j}(x-y)=0 .
$$

Accordingly, $q_{j}(B(x-y))=0$, i.e., $p_{j}\left(Q_{j} B(x-y)\right)=0$. As $p_{j}$ is a norm on $X_{j}$, we conclude via (4.19) that $B_{j} Q_{j}(x-y)=0$, i.e., $B_{j} Q_{j} x=B_{j} Q_{j} y$. So, the operator $\left(B_{j}, D\left(A_{j}\right)\right)$ is well defined on $X_{j}$. The linearity of $B_{j}$ easily follows by (4.19), the linearity of $B$ and of $Q_{j}$ and the surjectivity of $Q_{j}$.

The operator $\left(B_{j}, D\left(A_{j}\right)\right)$ is also $p_{j}$-dissipative on $X_{j}$. To see this, fix $\hat{x} \in D\left(A_{j}\right)$ and so $\hat{x}=Q_{j} x$ for some $x \in D(A)$ (as $D\left(A_{j}\right)=Q_{j}(D(A))$ ). Since $(B, D(B))$ is $\Gamma$-dissipative and $D(A) \subseteq D(B)$, by Proposition 4.2 we can find $x^{\prime} \in d q_{j}(x)$ such that $\operatorname{Re}\left\langle B x, x^{\prime}\right\rangle \leq 0$. Now, define a functional $\hat{x}^{\prime}$ on $X_{j}$ by

$$
\hat{x}^{\prime}\left(Q_{j} y\right):=x^{\prime}(y), \quad x \in X .
$$

The functional $\hat{x}^{\prime}$ is well defined and linear. Indeed, if $Q_{j} y=Q_{j} z$ for some $y, z \in X$, then $Q_{j}(y-z)=0$ and so $q_{j}(x-y)=0$. But, $x^{\prime} \in d q_{j}(x)$ and so $\left|\left\langle w, x^{\prime}\right\rangle\right| \leq q_{j}(w)$ for all $w \in X$. Thus, $\left|\left\langle y-z, x^{\prime}\right\rangle\right| \leq$ $q_{j}(y-z)=0$ and hence, $x^{\prime}(y)=x^{\prime}(z)$. The linearity of $\hat{x}^{\prime}$ follows easily. Moreover, $\hat{x}^{\prime} \in X_{j}^{\prime}$ because by (4.20) we have

$$
\left|\hat{x}^{\prime}(\hat{y})\right|=\left|\hat{x}^{\prime}\left(Q_{j} y\right)\right|=\left|x^{\prime}(y)\right| \leq q_{j}(y)=p_{j}\left(Q_{j} y\right),
$$

for all $\hat{y} \in X_{j}$ and $y \in X$ with $Q_{j} y=\hat{y}$. It follows that

$$
\left|\hat{x}^{\prime}(\hat{y})\right| \leq p_{j}(\hat{y}), \quad \hat{y} \in X_{j} .
$$

Now, we claim $\hat{x}^{\prime} \in d p_{j}(\hat{x})$. To see this, we first observe that $\operatorname{Re}\left\langle Q_{j} y, \hat{x}^{\prime}\right\rangle=\operatorname{Re}\left\langle y, x^{\prime}\right\rangle \leq q_{j}(y)=$ $p_{j}\left(Q_{j} y\right)$ for all $y \in X$. Therefore $\operatorname{Re}\left\langle\hat{y}, \hat{x}^{\prime}\right\rangle \leq p_{j}(\hat{y})$ for all $\hat{y} \in X_{j}$, being $Q_{j}$ surjective. We also have $\left\langle\hat{x}, \hat{x}^{\prime}\right\rangle=\left\langle x, x^{\prime}\right\rangle=q_{j}(x)=p_{j}(\hat{x})$. This proves the claim.

We now observe that from $\operatorname{Re}\left\langle B x, x^{\prime}\right\rangle \leq 0$ it follows by (4.19) that $\operatorname{Re}\left\langle B_{j} \hat{x}, \hat{x}^{\prime}\right\rangle=\operatorname{Re}\left\langle Q_{j} B x, \hat{x}^{\prime}\right\rangle=$ $\operatorname{Re}\left\langle B x, \hat{x}^{\prime} \circ Q_{j}\right\rangle=\operatorname{Re}\left\langle B x, x^{\prime}\right\rangle \leq 0$. Therefore, as $\hat{x} \in D\left(A_{j}\right)$ is arbitrary, we can conclude that $\left(B_{j}, D\left(A_{j}\right)\right)$ is $p_{j}$-dissipative on $X_{j}$.

The operator $\left(B_{j}, D\left(A_{j}\right)\right)$ is also $\left(p_{j}, A_{j}\right)$-bounded with $a_{p_{j}, 0}<1$. Indeed, since $(B, D(B))$ is $(\Gamma, A)$ bounded, we have

$$
q_{j}(B x) \leq a_{q_{j}} q_{j}(A x)+b_{q_{j}} q_{j}(x), \quad x \in D(A),
$$

for some positive constants $a_{q_{j}}, b_{q_{j}}>0$. So, (4.19) and (4.17) and the fact that $D\left(A_{j}\right)=Q_{j}(D(A))$ imply that

$$
\begin{aligned}
p_{j}\left(B_{j} Q_{j} x\right) & =p_{j}\left(Q_{j} B x\right)=q_{j}(B x) \leq a_{q_{j}} q_{j}(A x)+b_{q_{j}} q_{j}(x) \\
& =a_{q_{j}} p_{j}\left(Q_{j} A x\right)+b_{q_{j}} p_{j}\left(Q_{j} x\right)=a_{q_{j}} p_{j}\left(A_{j} Q_{j} x\right)+b_{q_{j}} p_{j}\left(Q_{j} x\right), \quad x \in D(A) .
\end{aligned}
$$


Hence, $a_{p_{j}, 0}=a_{q_{j}, 0}<1$.

As $\left(A_{j}, D\left(A_{j}\right)\right)$ is the infinitesimal generator of a $p_{j}$-contractive $C_{0}$-semigroup on $X_{j}$ and $\left(B_{j}, D\left(A_{j}\right)\right)$ is a $p_{j}$-dissipative, $\left(p_{j}, A_{j}\right)$-bounded operator with $a_{p_{j}, 0}<1$, the operator $\left(A_{j}+B_{j}, D\left(A_{j}\right)\right)$ generates a $p_{j}$-contractive $C_{0}$-semigroup $\left(S_{j}(t)\right)_{t \geq 0}$ on $X_{j}$; see [13, Theorem III.2.7]. Accordingly, $(0, \infty) \subseteq$ $\rho\left(A_{j}+B_{j}\right)$.

Since $j \in \mathbb{N}$ is arbitrary, we can see that $(0, \infty) \subseteq \rho(A+B)$. In fact, if $\lambda>0$, to conclude that $\lambda \in \rho(A+B)$ we need to show only that $(\lambda I-(A+B)): D(A) \subseteq X \rightarrow X$ is surjective. To do so, we observe that from (4.13), (4.17) and (4.19) it follows, for every $j \in \mathbb{N}$, that

$$
\begin{aligned}
& \left(\lambda I-\left(A_{j}+B_{j}\right)\right) Q_{j, j+1} Q_{j+1} x=\left(\lambda I-\left(A_{j}+B_{j}\right)\right) Q_{j} x=Q_{j}(\lambda I-(A+B)) x \\
& =Q_{j, j+1} Q_{j+1}(\lambda I-(A+B)) x=Q_{j, j+1}\left(\lambda I-\left(A_{j+1}+B_{j+1}\right)\right) Q_{j+1} x, \quad x \in D(A),
\end{aligned}
$$

and hence, as $D\left(A_{j}\right)=Q_{j}(D(A))=Q_{j, j+1} Q_{j+1}(D(A))=Q_{j, j+1}\left(D\left(A_{j+1}\right)\right)$, that

$$
\left(\lambda I-\left(A_{j}+B_{j}\right)\right) Q_{j, j+1} x=Q_{j, j+1}\left(\lambda I-\left(A_{j+1}+B_{j+1}\right) x, \quad x \in D\left(A_{j+1}\right) .\right.
$$

Now, fix $y \in X$. Then, for every $j \in \mathbb{N}$ there exists $x_{j} \in D\left(A_{j}\right)$ such that $\left(\lambda I-\left(A_{j}+B_{j}\right)\right) x_{j}=Q_{j} y$. Define $x:=\left(x_{j}\right)_{j \in \mathbb{N}} \in \prod_{j \in \mathbb{N}} X_{j}$. So, for every $j \in \mathbb{N}$ we get via (4.21) and (4.13) that

$$
\left(\lambda I-\left(A_{j}+B_{j}\right)\right) Q_{j, j+1} x_{j+1}=Q_{j, j+1}\left(\lambda I-\left(A_{j+1}+B_{j+1}\right)\right) x_{j+1}=Q_{j, j+1} Q_{j+1} y=Q_{j} y
$$

and hence, by the injectivity of $\left(\lambda I-\left(A_{j}+B_{j}\right)\right)$, we deduce that

$$
Q_{j, j+1} x_{j+1}=x_{j} .
$$

Since $X=\operatorname{proj}_{j}\left(X_{j}, Q_{j, j+1}\right)=\left\{\left(z_{j}\right)_{j \in \mathbb{N}} \in \prod_{j \in \mathbb{N}} X_{j}: Q_{j, j+1} z_{j+1}=z_{j} \forall j \in \mathbb{N}\right\}$ we can conclude that $x \in X$. Actually, $x \in D(A)$. This easily follows from the fact that $Q_{j} x=x_{j} \in D\left(A_{j}\right)$ for all $j \in \mathbb{N}$ and so, by (4.15), for every $j \in \mathbb{N}$ there exists in $X_{j}$ the limit

$$
\lim _{t \rightarrow 0^{+}} \frac{Q_{j} T(t) x-Q_{j} x}{t}=\lim _{t \rightarrow 0^{+}} \frac{T_{j}(t) x_{j}-x_{j}}{t}=A_{j} x_{j}, \quad j \in \mathbb{N} .
$$

So, there exists $\lim _{t \rightarrow 0^{+}} \frac{T(t) x-x}{t}$ in $X$ and $x \in D(A)$.

Moreover, $(\lambda I-(A+B)) x=y$ because $Q_{j}(\lambda I-(A+B)) x=\left(\lambda I-\left(A_{j}+B_{j}\right)\right) Q_{j} x=\left(\lambda I-\left(A_{j}+\right.\right.$ $\left.B_{j}\right) x_{j}=Q_{j} y$ for all $j \in \mathbb{N}$. This shows that $(\lambda I-(A+B)): D(A) \subseteq X \rightarrow X$ is surjective and so the proof is complete.

Example 4.13. We recall that the space $X$ given in Example 4.6 is a quojection. In particular, the space $X$ and the operators $(A, D(A))$ and $(B, X)$ satisfy all the assumptions of Theorem 4.12 with respect to $\Gamma=\left\{p_{h}\right\}_{h \in \mathbb{N}}$. In particular, the operator $(B, D(A))$ is $(\Gamma, A)$-bounded with bound $a_{p_{h}, 0}=0<1$ for all $h \in \mathbb{N}$, being $B \in \mathcal{L}(X)$. Then by Theorem 4.12 we can immediately conclude that the operator $(A+B, D(A))$ generates a $\Gamma$-contractively equicontinuous $C_{0}$-semigroup $(T(t))_{t \geq 0}$ on $X$ without checking that the equality in (4.4) is satisfied for some $\lambda>0$.

In case the bounds $a_{p_{j}, 0}=1$, for $j \in \mathbb{N}$, the result continues to hold under the assumptions either of Theorem 4.10 or of Theorem 4.12 whenever the dual operator of $(B, D(B))$ is also densely defined in $X_{\beta}$ exactly as in the Banach space setting, as the following proposition shows; for the Banach space case, see, for example, [13, Corollary III.2.8].

Proposition 4.14. Let $X$ be a Fréchet space satisfying the assumptions either of Theorem 4.10 or of Theorem 4.12 with respect to $\Gamma=\left\{p_{j}\right\}_{j \in \mathbb{N}} \subseteq \Gamma_{X}$. Let $(A, D(A))$ be the infinitesimal generator of a $\Gamma$-contractively equicontinuous $C_{0}$-semigroup on $X$. Let $(B, D(B))$ be a $\Gamma$-dissipative, $(\Gamma, A)$-bounded operator on $X$ such that

$$
p_{j}(B x) \leq p_{j}(A x)+b_{j} p_{j}(x), \quad x \in D(A), j \in \mathbb{N},
$$

for some positive constant $b_{j}$, for $j \in \mathbb{N}$. If $\left(B^{\prime}, D\left(B^{\prime}\right)\right)$ is densely defined on $X_{\beta}^{\prime}$, then the closure of $(A+B, D(A))$ generates a $\Gamma$-contractively equicontinuous $C_{0}$-semigroup on $X$. 
Proof. Under such assumptions the operator $(A+B, D(A))$ is densely defined and $\Gamma$-dissipative. Hence, by Theorem 3.13 it suffices to show that $\operatorname{rg}(I-(A+B))$ is dense in $X$.

Assume that $\operatorname{rg}(I-(A+B))$ is not dense in $X$. Then there exists $y^{\prime} \in X^{\prime} \backslash\{0\}$ such that $\left\langle z, y^{\prime}\right\rangle=0$ for all $z \in \operatorname{rg}(I-(A+B))$. Since $y^{\prime} \in X^{\prime} \backslash\{0\}$ there exists $k \in \mathbb{N}$ such that $p_{k}^{\prime}\left(y^{\prime}\right) \neq 0$ and so we can choose $y \in X$ satisfying $\left\langle y, y^{\prime}\right\rangle=p_{k}^{\prime}\left(y^{\prime}\right)$.

Now, fix $\varepsilon \in(0,1)$ and observe that $(\varepsilon B, D(B))$ is also $\Gamma$-dissipative and $(\Gamma, A)$-bounded with $a_{p_{j}, 0}<1$ for all $j \in \mathbb{N}$. Indeed, for a fixed $j \in \mathbb{N}$, we have that

$$
p_{j}((\lambda-\varepsilon B) x)=\varepsilon p_{j}\left(\left(\varepsilon^{-1} \lambda-B\right) x\right) \geq \varepsilon\left(\varepsilon^{-1} \lambda p_{j}(x)\right)=\lambda p_{j}(x), \quad x \in D(B),
$$

and by (4.22) that

$$
p_{j}(\varepsilon B x)=p_{j}(B(\varepsilon x)) \leq p_{j}(A(\varepsilon x))+b_{j} p_{j}(\varepsilon x)=\varepsilon p_{j}(A x)+\varepsilon b_{j} p_{j}(x), \quad x \in D(A),
$$

and so $a_{p_{j}, 0}<1$. Accordingly to Theorem 4.10 or to Theorem 4.12 , the operator $(A+\varepsilon B, D(A))$ generates a $\Gamma$-contractively equicontinuous $C_{0}$-semigroup on $X$. So, $1 \in \rho(A+\varepsilon B)$ and hence, there exists $x_{\varepsilon} \in D(A)$ satisfying $x_{\varepsilon}-(A+\varepsilon B) x_{\varepsilon}=y$ and $p_{j}\left(x_{\varepsilon}\right) \leq p_{j}(y)$ for all $j \in \mathbb{N}$ (because $A+\varepsilon B$ is also $\Gamma$-dissipative). Moreover, by (4.22) we also have

$$
\begin{aligned}
p_{j}\left(B x_{\varepsilon}\right) & \leq p_{j}\left(A x_{\varepsilon}\right)+b_{j} p_{j}\left(x_{\varepsilon}\right) \\
& \leq p_{j}\left((A+\varepsilon B) x_{\varepsilon}\right)+\varepsilon p_{j}\left(B x_{\varepsilon}\right)+b_{j} p_{j}\left(x_{\varepsilon}\right) \\
& =p_{j}\left(x_{\varepsilon}-y\right)+\varepsilon p_{j}\left(B x_{\varepsilon}\right)+b_{j} p_{j}\left(x_{\varepsilon}\right), \quad j \in \mathbb{N},
\end{aligned}
$$

and hence,

$$
(1-\varepsilon) p_{j}\left(B x_{\varepsilon}\right) \leq p_{j}\left(x_{\varepsilon}-y\right)+b_{j} p_{j}\left(x_{\varepsilon}\right) \leq\left(2+b_{j}\right) p_{j}(y) .
$$

If $z^{\prime} \in D\left(B^{\prime}\right)$ and so $p_{l}^{\prime}\left(B z^{\prime}\right)<\infty$ for some $l \in \mathbb{N}$, then it follows that

$$
\left|\left\langle(1-\varepsilon) B x_{\varepsilon}, z^{\prime}\right\rangle\right| \leq(1-\varepsilon) p_{l}\left(x_{\varepsilon}\right) p_{l}^{\prime}\left(B^{\prime} z^{\prime}\right) \leq(1-\varepsilon) p_{l}(y) p_{l}^{\prime}\left(B^{\prime} z^{\prime}\right) .
$$

Since $\varepsilon \in(0,1)$ is arbitrary, we can let $\varepsilon \rightarrow 1^{-}$in (4.24), to obtain

$$
\lim _{\varepsilon \rightarrow 1^{-}}\left\langle(1-\varepsilon) B x_{\varepsilon}, z^{\prime}\right\rangle=0
$$

Since $\left\{(1-\varepsilon) p_{j}\left(B x_{\varepsilon}\right): \varepsilon \in(0,1)\right\} \in \mathcal{B}(X)$ thanks to $(4.23)$ and $D\left(B^{\prime}\right)$ is dense in $X_{\beta}^{\prime}$, it follows that

$$
\lim _{\varepsilon \rightarrow 1^{-}}\left\langle(1-\varepsilon) B x_{\varepsilon}, y^{\prime}\right\rangle=0
$$

and so

$$
\begin{aligned}
p_{k}^{\prime}\left(y^{\prime}\right) & =\left\langle y, y^{\prime}\right\rangle=\left\langle x_{\varepsilon}-(A+\varepsilon B) x_{\varepsilon}, y^{\prime}\right\rangle=\left\langle(1-\varepsilon) B x_{\varepsilon}, y^{\prime}\right\rangle+\left\langle(I-(A+B)) x_{\varepsilon}, y^{\prime}\right\rangle \\
& =\left\langle(1-\varepsilon) B x_{\varepsilon}, y^{\prime}\right\rangle \rightarrow 0 \text { as } \varepsilon \rightarrow 1^{-}
\end{aligned}
$$

this is a contradiction as $p_{k}^{\prime}\left(y^{\prime}\right) \neq 0$. Therefore, $\operatorname{rg}(I-(A+B))$ is dense in $X$ and the proof is complete.

If $X$ is a reflexive lcHs, then the dual operator of every closable, densely defined operator on $X$ is densely defined on $X_{\beta}^{\prime}$; see Proposition 3.7(ii). On the other hand, dissipative, densely defined operators are always closable; see Proposition 3.12. So, we easily obtain thanks to Proposition 4.14 the following result.

Corollary 4.15. Let $X$ be a reflexive Fréchet space satisfying the assumptions either of Theorem 4.10 or of Theorem 4.12 with respect to $\Gamma \subseteq \Gamma_{X}$. Let $(A, D(A))$ be the infinitesimal generator of a $\Gamma$-contractively equicontinuous $C_{0}$-semigroup on $X$. If $(B, D(B))$ is a $\Gamma$-dissipative, $(\Gamma, A)$-bounded operator on $X$ satisfying the inequalities in (4.22), then the closure of $(A+B, D(A))$ generates a $\Gamma$-contractively equicontinuous $C_{0}$-semigroup on $X$. 


\section{Additive perturbations and (uniformly) mean ergodic operators}

The results in Sections 2 and 3 together with recent developments in ergodic theory in lcHs' investigated in $[1,2,6,8]$ allow us to extend to this setting some results on additive perturbations on Banach spaces in terms of the ergodic properties of the operator $B R(\lambda, A)$ due to Tyran-Kamińska [26].

First we recall some necessary notation and facts. Given any lcHs $X$ and $T \in \mathcal{L}(X)$, let us introduce the notation

$$
T_{[n]}:=\frac{1}{n} \sum_{m=1}^{n} T^{m}, \quad n \in \mathbb{N},
$$

for the Cesàro means of $T$. Then $T$ is called mean ergodic precisely when $\left\{T_{[n]}\right\}_{n=1}^{\infty}$ is a convergent sequence in $\mathcal{L}_{s}(X)$. If $\left\{T_{[n]}\right\}_{n=0}^{\infty}$ happens to be convergent in $\mathcal{L}_{b}(X)$, then $T$ will be called uniformly mean ergodic.

We always have the identities

$$
(I-T) T_{[n]}=T_{[n]}(I-T)=\frac{1}{n}\left(T-T^{n+1}\right), \quad n \in \mathbb{N},
$$

and also (setting $T_{[0]}:=I$ ) that

$$
\frac{1}{n} T^{n}=T_{[n]}-\frac{(n-1)}{n} T_{[n-1]}, \quad n \in \mathbb{N} .
$$

If $T$ is power bounded, then

$$
\overline{\operatorname{rg}(I-T)}=\left\{x \in X: \lim _{n \rightarrow \infty} T_{[n]} x=0\right\}, \overline{\operatorname{rg}(I-T)} \cap \operatorname{Ker}(I-T)=\{0\}
$$

and such a $T$ clearly satisfies $\frac{1}{n} T^{n} \rightarrow 0$ in $\mathcal{L}_{b}(X)$ (hence, in $\left.\mathcal{L}_{s}(X)\right)$ for $n \rightarrow \infty$.

If $T$ is mean ergodic (uniformly mean ergodic, resp.), then the identity in (5.3) implies that $\frac{1}{n} T^{n} \rightarrow 0$ in $\mathcal{L}_{s}(X)$ for $n \rightarrow \infty$ (in $\mathcal{L}_{b}(X)$, resp.). So, if, in addition, $X$ is barrelled, then the identities in (5.4) are satisfied and we also have

$$
X=\operatorname{Ker}(I-T) \oplus \overline{\operatorname{rg}(I-T)} \text {; }
$$

see [2, Propositions 2.1 and 2.2] (or see [1]).

Theorem 5.1. Let $X$ be a complete, barrelled lcHs. Let $(A, D(A))$ be a densely defined operator on $X$ such that $\rho(A)_{+}:=\rho(A) \cap(0, \infty) \neq \emptyset$. Let $(B, D(B))$ be an operator on $X$ such that $D(A) \subseteq D(B)$, $(A+B, D(A))$ is dissipative and $B R(\lambda, A) \in \mathcal{L}(X)$ is mean ergodic for some $\lambda \in \rho(A)_{+}$. Then the closure of $(A+B, D(A))$ generates an equicontinuous $C_{0}$-semigroup on $X$.

Proof. Let $\lambda \in \rho(A)_{+}$such that $B R(\lambda, A) \in \mathcal{L}(X)$ is mean ergodic. Then

$$
X=\operatorname{Ker}(I-B R(\lambda, A)) \oplus \overline{\operatorname{rg}(I-B R(\lambda, A))} .
$$

But, $\operatorname{Ker}(I-B R(\lambda, A))=\{0\}$ and so $\overline{\operatorname{rg}(I-B R(\lambda, A))}=X$. To see this, we observe that

$$
(\lambda I-(A+B)) x=(I-B R(\lambda, A))(\lambda I-A) x, \quad x \in D(A),
$$

where $(\lambda I-A)(D(A))=X$. If $(I-B R(\lambda, A)) y=0$ for some $y \in X$, then there exists $x \in D(A)$ such that $y=(\lambda I-A) x$ and hence

$$
(\lambda I-(A+B)) x=(I-B R(\lambda, A))(\lambda I-A) x=(I-B R(\lambda, A)) y=0 .
$$

Since $(A+B, D(A))$ is dissipative and so injective by Proposition 3.10(i), it follows that $x=0$. Consequently, $y=(\lambda I-A) x=0$. Then, $\operatorname{Ker}(I-B R(\lambda, A))=\{0\}$.

The fact that $\overline{\operatorname{rg}(I-B R(\lambda, A))}=X$ implies from Remark 4.7(ii) that $\overline{\operatorname{rg}(\lambda I-(A+B))}=X$.

Since $(A+B, D(A))$ is dissipative and $\overline{\operatorname{rg}(\lambda I-(A+B))}=X$, we can conclude thanks to Theorem 3.13 that the closure of $(A+B, D(A))$ generates an equicontinuous $C_{0}$-semigroup on $X$.

Conversely, the following fact holds. 
Theorem 5.2. Let $X$ be a complete, barrelled lcHs. Let $(A, D(A))$ and $(B, D(B))$ be two operators on $X$ such that $D(A) \subseteq D(B)$. Let us assume that the closure of $(A+B, D(A))$ generates an equicontinuous $C_{0}$-semigroup on $X$ and $B R(\lambda, A) \in \mathcal{L}(X)$ is power bounded for some $\lambda \in \rho(A)_{+}$. Then the operator $B R(\lambda, A)$ is mean ergodic.

Proof. Since the closure of $(A+B, D(A))$ generates an equicontinuous $C_{0}$-semigroup on $X$, the operator $(A+B, D(A))$ is dissipative and densely defined; see Remark 3.11. It follows from Proposition 3.12 that

$$
\overline{\operatorname{rg}(\mu I-(A+B))}=\operatorname{rg}(\mu I-\overline{A+B})=X, \quad \mu>0 .
$$

Hence, $\overline{\operatorname{rg}(I-B R(\lambda, A))}=\overline{\operatorname{rg}(\lambda I-(A+B))}=X$. This fact together with the power boundedness of $B R(\lambda, A)$ imply by $(5.4)$ that $B R(\lambda, A)$ is mean ergodic.

If $X$ is a reflexive lcHs in which every relatively $\sigma\left(X, X^{\prime}\right)$-compact subset is also relatively sequentially $\sigma\left(X, X^{\prime}\right)$-compact, then every power bounded operator $T \in \mathcal{L}(X)$ is mean ergodic; see [1, Corollary 2.7] and [2, Proposition 2.3]. So, by Theorem 5.1 we immediately obtain the following result.

Corollary 5.3. Let $X$ be a complete lcHs. Let $(A, D(A))$ and $(B, D(A))$ be operators on $X$. Suppose that $(A+B, D(A))$ is a dissipative, densely defined operator on $X$ and that $B R(\lambda, A) \in \mathcal{L}(X)$ is power bounded for some $\lambda \in \rho(A)_{+}$. If $X$ is a reflexive lcHs in which every relatively $\sigma\left(X, X^{\prime}\right)$-compact subset is also relatively sequentially $\sigma\left(X, X^{\prime}\right)$-compact, then the closure of $(A+B, D(A))$ generates an equicontinuous $C_{0}$-semigroup on $X$.

In case the space $X$ is a quojection Fréchet space, the following results are also available.

Theorem 5.4. Let $X$ be a quojection Fréchet space. Let $(A, D(A))$ be a densely defined operator on $X$ such that $\rho(A)_{+}:=\rho(A) \cap(0, \infty) \neq \emptyset$. Let $(B, D(B))$ be an operator on $X$ such that $D(A) \subseteq D(B)$, $(A+B, D(A))$ is dissipative and $B R(\lambda, A) \in \mathcal{L}(X)$ is uniformly mean ergodic for some $\lambda \in \rho(A)_{+}$. Then $(A+B, D(A))$ generates an equicontinuous $C_{0}$-semigroup on $X$.

Proof. By Theorem 5.1 the closure of the operator $(A+B, D(A))$ generates an equicontinuous $C_{0^{-}}$ semigroup on $X$. Accordingly, $\overline{\operatorname{rg}(\mu I-(A+B))}=X$ for every $\mu>0$.

Since $B R(\lambda, A) \in \mathcal{L}(X)$ is uniformly mean ergodic, then $\operatorname{rg}(I-B R(\lambda, A))$ is a closed subspace of $X$; see [6, Theorem 3.5]. But, $\operatorname{rg}(\lambda I-(A+B))=\operatorname{rg}(I-B R(\lambda, A))$; see (4.9). This identity implies that $\operatorname{rg}(\lambda I-(A+B))=X$ and so that the operator $(A+B, D(A))$ is closed, being $(A+B, D(A))$ dissipative; see Proposition 3.10(ii). This completes the proof.

Conversely, we have

Theorem 5.5. Let $X$ be a quojection Fréchet space. Let $(A, D(A))$ and $(B, D(B))$ be two operators on $X$ such that $D(A) \subseteq D(B)$. Let us assume that the operator $(A+B, D(A))$ is the infinitesimal generator of an equicontinuous $C_{0}$-semigroup on $X$ and $B R(\lambda, A) \in \mathcal{L}(X)$ is power bounded for some $\lambda \in \rho(A)_{+}$. Then $B R(\lambda, A)$ is uniformly mean ergodic.

Proof. By Theorem 5.2 we can conclude that $B R(\lambda, A)$ is mean ergodic. On the other hand, the assumptions ensure that $(A+B, D(A))$ is a dissipative, closed operator on $X$. So, by Proposition 3.10(ii) the subspace $\operatorname{rg}(\lambda I-(A+B))$ is then closed in $X$ and hence, also $\operatorname{rg}(I-B R(\lambda, A))$ is a closed subspace of $X$. This implies via Theorem 3.5 of $[6]$ that $B R(\lambda, A)$ is uniformly mean ergodic.

Let $X$ be a lcHs. An operator $T \in \mathcal{L}(X)$ is called Montel if $T$ maps bounded subsets of $X$ into relatively compact subsets of $X$. In case $X$ is a Banach space, if $T$ is Montel, then it is clearly a compact operator on $X$. While, an operator $T \in \mathcal{L}(X)$ is called quasi-Montel if there exists a sequence $\left\{M_{n}\right\}_{n \in \mathbb{N}} \subseteq \mathcal{L}(X)$ of Montel operators such that $\left(T-M_{n}\right) \rightarrow 0$ in $\mathcal{L}_{b}(X)$ as $n \rightarrow \infty$. In case $X$ is a Banach space, if $T$ is quasi-Montel, then it is a quasi-compact operator on $X$. Now, Theorem 5.4 combined with Theorem 35 of [8] yields the following result.

Corollary 5.6. Let $X$ be a quojection Fréchet space. Let $(A, D(A))$ and $(B, D(A))$ be two operators on $X$ such that $D(A) \subseteq D(B)$. Suppose that $(A+B, D(A))$ is a dissipative, densely defined operator on $X$ and that $B R(\lambda, A) \in \mathcal{L}(X)$ is power bounded for some $\lambda \in \rho(A)_{+}$. If $B R(\lambda, A) \in \mathcal{L}(X)$ is quasi-Montel, then $(A+B, D(A))$ generates an equicontinuous $C_{0}$-semigroup on $X$. 
The next example shows that the result stated in Theorem 5.4 remains confined to the setting of quojection Fréchet spaces.

Example 5.7. An increasing sequence $A=\left(a_{j}\right)_{j}$ of functions $a_{j}: \mathbb{N} \rightarrow[0, \infty)$ is called a Köthe matrix on $\mathbb{N}$, where by increasing we mean $0 \leq a_{j}(i) \leq a_{j+1}(i)$ for all $i, j \in \mathbb{N}$. For each $p \in[1, \infty)$ let

$$
\lambda_{p}(A):=\left\{x \in \mathbb{C}^{\mathbb{N}}: q_{j}^{(p)}(x):=\left(\sum_{i \in \mathbb{N}}\left|a_{j}(i) x_{i}\right|^{p}\right)^{1 / p}<\infty, \quad \forall j \in \mathbb{N}\right\} .
$$

Elements $x \in \mathbb{C}^{\mathbb{N}}$ are denoted by $x=\left(x_{i}\right)_{i}$. The spaces $\lambda_{p}(A)$, for $p \in[1, \infty]$, are called Köthe echelon spaces (of order $p$ ); they are all Fréchet spaces (separable and reflexive if $p \neq 1$ ) relative to the increasing sequence of seminorms $\left\{q_{j}^{(p)}\right\}_{j \in \mathbb{N}}$.

Fix $1 \leq p<\infty$ and $\mu=\left(\mu_{i}\right)_{i} \subseteq \mathbb{R}^{+}$. For each $t \geq 0$ set $T(t) x:=\left(e^{-\mu_{i} t} x_{i}\right)_{i}$ for $x \in \lambda_{p}(A)$. Then it is easy to verify that $(T(t))_{t \geq 0} \subseteq \mathcal{L}\left(\lambda_{p}(A)\right)$ is an equicontinuous $C_{0}$-semigroup on $\lambda_{p}(A)$ whose infinitesimal generator $(A, D(A))$ is given by

$$
A x:=\left(-\mu_{i} x_{i}\right)_{i}, \quad x \in D(A):=\left\{x \in \lambda_{p}(A): \mu \cdot x=\left(\mu_{i} x_{i}\right)_{i} \in \lambda_{p}(A)\right\} .
$$

In particular, $(A, D(A))$ is a dissipative, densely defined closed operator on $\lambda_{p}(A)$; see Remark 3.11.

If $\lambda_{p}(A) \neq \mathbb{C}^{\mathbb{N}}$ and Montel, there exists $\left(d_{i}\right)_{i} \subseteq \mathbb{R}^{+}$with $0<d_{i}<1$ for $i \in \mathbb{N}$ such that the diagonal operator $S x:=\left(d_{i} x_{i}\right)_{i}$ for $x \in \lambda_{p}(A)$ is power bounded (i.e., $q_{j}^{(p)}\left(S^{k} x\right) \leq q_{j}^{(p)}(x)$ for all $k, j \in \mathbb{N}$ and $\left.x \in \lambda_{p}(A)\right)$, uniformly mean ergodic but, $(I-S)\left(\lambda_{p}(A)\right)$ is a not closed, dense subspace of $\lambda_{p}(A)$; see [6, Proposition 3.1].

Now, we choose $\left(\mu_{i}\right)_{i} \subseteq \mathbb{R}^{+}$and $\lambda>0$ so that $\frac{\mu_{i}}{\lambda}>\frac{d_{i}}{1-d_{i}}$ for all $i \in \mathbb{N}$ and define the operator $B:=S(\lambda-A): D(A) \subseteq \lambda_{p}(A) \rightarrow \lambda_{p}(A)$. Then $B R(\lambda, A)=S$ is a power bounded, uniformly mean ergodic operator on $\lambda_{p}(A)$. Moreover, we have

$$
(\mu-(A+B)) x=\left(\left(\mu+\left(1-d_{i}\right) \mu_{i}-d_{i} \lambda\right) x_{i}\right)_{i}, \quad x \in D(A), \mu>0 .
$$

Since $\frac{\mu_{i}}{\lambda}>\frac{d_{i}}{1-d_{i}}$ for all $i \in \mathbb{N}$ and hence, $\left(1-d_{i}\right) \mu_{i}-d_{i} \lambda>0$ for all $i \in \mathbb{N}$, it follows, for every $j \in \mathbb{N}$ and $x \in D(A)$, that

$$
\left[q_{j}^{(p)}((\mu-(A+B)) x)\right]^{p}=\sum_{i \in \mathbb{N}}\left|a_{j}(i)\left(\mu+\left(1-d_{i}\right) \mu_{i}-d_{i} \lambda\right) x_{i}\right|^{p} \geq \mu^{p} \sum_{i \in \mathbb{N}}\left|a_{j}(i) x_{i}\right|^{p}=\mu^{p}\left[q_{j}^{(p)}(x)\right]^{p} .
$$

Therefore, the operator $(A+B, D(A))$ is dissipative.

On the other hand, we have

$$
\lambda-(A+B)=\lambda-A-S(\lambda-A)=(I-S)(\lambda-A) \quad \text { on } D(A),
$$

from which it follows

$$
(\lambda-(A+B))(D(A))=(I-S)(\lambda-A)(D(A))=(I-S)\left(\lambda_{p}(A)\right) .
$$

So, $(\lambda-(A+B))(D(A))$ is a non closed dense subspace of $\lambda_{p}(A)$.

We can then apply Theorem 5.1 to conclude that the closure of the operator $(A+B, D(A))$ generates an equicontinuous $C_{0}$-semigroup on $\lambda_{p}(A)$. But, the operator $(A+B, D(A))$ is not closed, being $(\lambda-(A+B))(D(A))$ a non closed subspace of $\lambda_{p}(A)$.

We conclude this section with the following fact which is the quojection version of Lemma 2.1 of [21].

Proposition 5.8. Let $X$ be a quojection Fréchet space and let $T \in \mathcal{L}(X)$ satisfy $\frac{T^{n}}{n} \rightarrow 0$ in $\mathcal{L}_{b}(X)$ for $n \rightarrow \infty$. Suppose that $T$ is mean ergodic but, not uniformly mean ergodic. Then $T$ cannot be dissipative.

Proof. Suppose that $T$ is dissipative. Then $\operatorname{Ker}(I-T)=\{0\}$. So, as $T$ is mean ergodic, it follows that $X=\overline{\operatorname{rg}(I-T)}$. But, $T$ is not uniformly ergodic and so $\operatorname{rg}(I-T)$ cannot be closed in $X ;[6$, Theorem 3.5]. Accordingly, $1 \notin \rho(T)$. This is a contradiction with the dissipativity of $T$. Indeed, if $T$ is dissipative, then $\mathbb{C}_{+} \subseteq \rho(T)$; see Corollary 3.15. 
Acknowledgements The research of the second author was partially supported by MINECO of Spain, Project MTM2013-43540-P and by Programa de Apoyo a la Investigación y Desarrollo de la UPV, PAID-06-12.

\section{References}

[1] A.A. Albanese, J. Bonet, W.J. Ricker, Mean ergodic operators in Fréchet spaces, Ann. Acad. Sci. Fenn. Math. 34 (2009), 401-436.

[2] A.A. Albanese, J. Bonet, W.J. Ricker, Mean ergodic operators, in: Vector Measures, Integration and Related Topics, G.P. Curbera et. al. (Eds), Operator Theory: Advances and Applications 201, Birkhäuser Verlag Basel, (2010), pp. 1-20.

[3] A.A. Albanese, J. Bonet, W.J. Ricker, $C_{0}$-semigroups and mean ergodic operators in a class of Fréchet spaces, J. Math. Anal. Appl. 365 (2010), 142-157.

[4] A.A. Albanese, J. Bonet, W.J. Ricker, Mean ergodic semigroups of operators, Rev. R. Acad. Cien. Serie A, Mat., RACSAM, 106 (2012), 299-319.

[5] A.A. Albanese, J. Bonet, W.J. Ricker, Montel resolvents and uniformly mean ergodic semigroups of linear operators, Quaest. Math. 36 (2013), 253-290.

[6] A.A. Albanese, J. Bonet, W.J. Ricker, Convergence of arithmetic means of operators in Fréchet spaces, J. Math. Anal. Appl. 401 (2013), 160-173.

[7] A.A. Albanese, J. Bonet, W.J. Ricker, Uniform mean ergodicity of $C_{0}$-semigroups in a class of Fréchet spaces, Funct. Approx. Comment. Math. 50, (2), (2014), 307-349.

[8] A.A. Albanese, J. Bonet, W.J. Ricker, Uniform Convergence and spectra of operators in a class of Fréchet spaces, Abstract Appl. Analysis, Volume 2014, Article ID 179027, 16 pages.

[9] W. Arendt, Contraction semigroups and dissipative operators, in: One-parameter semigroups of positive operators (ed. Nagel, R.), Lect. Notes in Math. 1184, Springer-Verlag, Berlin-Heidelberg, 1986, pp. 47-54.

[10] S.F. Bellenot, E. Dubinsky, Fréchet spaces with nuclear Köthe quotients, Trans. Amer. Math. Soc. 273 (1982), 579-594.

[11] J. Bonet, M. Maestre, G. Metafune, V.B. Moscatelli, D. Vogt, Every quojection is the quotient of a countable product of Banach spaces, in: "Advances in the Theory of Fréchet spaces", T. Terzioğlu (Ed.), NATO ASI Series, 287, Kluwer Acad. Publ., Dordrecht, 1989, pp. 355-356.

[12] P. Domański, M. Langenbruch, On the abstract Cauchy problem for operators in locally convex spaces, Rev. R. Acad. Cien. Serie A, Mat., RACSAM, 106 (2012), 247-273.

[13] K.-J. Engel, R. Nagel, One-parameter semigroups for linear evolution equations, Graduates Texts in Math. 194, Springer-Verlag, New York-Berlin, 2000.

[14] L. Frerick, E. Jordá, T. Kalmes, J. Wengenroth, Strongly continuous semigroups on some Fréchet spaces. J. Math. Anal. Appl. 412, (1), (2014), 121-124.

[15] B. Jacob, S.-A. Wegner, Asymptotics of solution equations beyond Banach spaces, Semigroup Forum, to appear.

[16] B. Jacob, S.-A. Wegner, J. Wintermayr, Desch-Schappacher perturbation of one-parameter semigroups on locally convex spaces, Math. Nachr., to appear.

[17] G. Köthe, Topological vector spaces I, 2nd Rev. Ed., Springer Verlag, Berlin-Heidelberg-New York, 1983.

[18] G. Köthe, Topological vector spaces II, Springer Verlag, Berlin-Heidelberg-New York, 1979.

[19] H. Komatsu, Semi-groups of operators in locally convex spaces, J.Math. Soc. Japan 16 (1964), 230-262.

[20] T. Komura, Semigroups of operators in locally convex spaces, J. Funct. Anal. 2 (1969), 258-296.

[21] G. Lumer, R.S. Phillips, Dissipative operators in a Banach space, Pacific J. Math., (2), 11, (1961) 679-698.

[22] G. Metafune, V.B. Moscatelli, Quojections and prequojections, in: "Advances in the Theory of Fréchet spaces", T. Terzioğlu (Ed.), NATO ASI Series, 287, Kluwer Academic Publishers, Dordrecht, 1989, pp. 235-254.

[23] I. Miyadera, Semigroups of operators in Fréchet spaces and applications to partial differential operators, Tôhoku Math. J. 11 (1959), 162-183.

[24] V.B. Moscatelli, Fréchet spaces without norms and without bases, Bull. London Math. Soc. 12 (1980), 63-66.

[25] A. Pazy, Semigroups of linear operators and applications to partial differential equations, Appl. Math. Sci. 44, Springer-Verlag, New York-Berlin, 1983.

[26] M. Tyran-Kamińska, Ergodic theorems and perturbations of contraction semigroups, Studia Math. 195 (2009), $147-155$

[27] S.-A. Wegner, The growth bound for strongly continuous semigroups on Fréchet spaces, Proc. Edinb. Math. Soc., to appear.

[28] K. Yosida, Functional analysis, Springer-Verlag, New York-Berlin, 1980. 
Dipartimento di Matematica e Fisica "E. De Giorgi", Università del Salento, Via per Arnesano, C.P. 193, 73100 LECCE, ITALY

E-mail address: angela.albanese@unisalento.it

Instituto Universitario de Matemática Pura y Aplicada iUmPA, Universitat Politècnica de València, Camino de Vera, s/n, E-46022 Valencia, Spain

E-mail address: djornet@mat.upv.es 